\title{
IL-1RA regulates immunopathogenesis during fungal-associated allergic airway inflammation
}

Matthew S. Godwin, ${ }^{1}$ Kristen M. Reeder, ${ }^{1}$ Jaleesa M. Garth, ${ }^{1}$ Jonathan P. Blackburn, ${ }^{1}$ MaryJane Jones, ${ }^{2}$ Zhihong Yu, ${ }^{3}$ Sadis Matalon, ${ }^{3}$ Annette T. Hastie, ${ }^{4}$ Deborah A. Meyers, ${ }^{5}$ and Chad Steele ${ }^{2}$

'Department of Medicine, University of Alabama at Birmingham (UAB), Birmingham, Alabama, USA. ${ }^{2}$ Department of Microbiology and Immunology, Tulane University, New Orleans, Louisiana, USA. ${ }^{3}$ Department of Anesthesiology, UAB, Birmingham, Alabama, USA. ${ }^{4}$ Department of Medicine, Wake Forest University, Winston-Salem, North Carolina, USA. ${ }^{5}$ Department of Medicine, University of Arizona, Tucson, Arizona, USA.

Severe asthma with fungal sensitization (SAFS) defines a subset of human asthmatics with allergy to 1 or more fungal species and difficult-to-control asthma. We have previously reported that human asthmatics sensitized to fungi have worse lung function and a higher degree of atopy, which was associated with higher IL-1 receptor antagonist (IL-1RA) levels in bronchoalveolar lavage fluid. IL-1RA further demonstrated a significant negative association with bronchial hyperresponsiveness to methacholine. Here, we show that IL-1 $\alpha$ and IL-1 $\beta$ are elevated in both bronchoalveolar lavage fluid and sputum from human asthmatics sensitized to fungi, implicating an association with IL-1 $\alpha$, IL-1 $\beta$, or IL-1RA in fungal asthma severity. In an experimental model of fungal-associated allergic airway inflammation, we demonstrate that IL-1R1 signaling promotes type 1 (IFN- $\gamma$, CXCL9, CXCL10) and type 17 (IL-17A, IL-22) responses that were associated with neutrophilic inflammation and increased airway hyperreactivity. Each of these were exacerbated in the absence of IL-1RA. Administration of human recombinant IL-1RA (Kineret/anakinra) during fungal-associated allergic airway inflammation improved airway hyperreactivity and lowered type 1 and type 17 responses. Taken together, these data suggest that IL-1R1 signaling contributes to fungal asthma severity via immunopathogenic type 1 and type 17 responses and can be targeted for improving allergic asthma severity.

Conflict of interest: The authors have declared that no conflict of interest exists.

Copyright: (c) 2019, American Society for Clinical Investigation.

Submitted: March 25, 2019 Accepted: September 19, 2019 Published: November 1, 2019

Reference information: JCI Insight 2019;4(21):e129055 https://doi.org/10.1172/jci. insight.129055.

\section{Introduction}

Atopic asthma is a chronic allergic disease wherein exposure to allergens results in a heterogeneous mixture of symptoms, including increased IgE, bronchiectasis, smooth muscle cell hypertrophy and hyperplasia, goblet cell hyperplasia, mucus hypersecretion, airway hyperresponsiveness (AHR) and remodeling, neutrophilia, eosinophilia, and lung fibrosis $(1,2)$. While there are numerous allergens associated with asthma exacerbations, fungi, including the mold Aspergillus fumigatus, are considered the most ubiquitous. Herein, a subset of asthma termed severe asthma with fungal sensitization (SAFS) has been described for individuals whose asthma is poorly controlled and who are sensitized to the fungal organisms Alternaria, Aspergillus, Cladosporium and/or Penicillium (3). Reports indicate that the estimated prevalence of SAFS ranges from $17 \%-46 \%$ of the estimated $5 \%-10 \%$ asthmatics defined as severe (4-7). As a result, individuals sensitive to fungi experience more frequent exacerbations, have higher incidences of uncontrolled asthma, and use corticosteroids more frequently than nonsensitive asthmatics (4-7).

A growing area of interest is the identification of factors that contribute to immunopathogenesis in allergic asthma and determining whether such factors could be viable therapeutic targets. Indeed, biologics targeting the type 2 cytokines IL-4, IL-5, and IL-13 have been successful in treating severe asthma (8). With regard to SAFS specifically, studies comparing genetic susceptibility in individuals with atopic asthma vs. individuals with SAFS have identified the pattern recognition receptors (PRRs) TLR3, TLR9, and Dectin-1 and the cytokines/chemokines IL-10, CCL2, and CCL17 as candidates for therapeutic development (9). We have also recently identified IL-7 as a potential immunopathogenic factor in human asthma and experimental fungalassociated allergic airway inflammation (10). In a study of pediatric asthmatics, those with SAFS demonstrated higher IgE levels and required higher usage of maintenance oral steroids to achieve similar clinical 

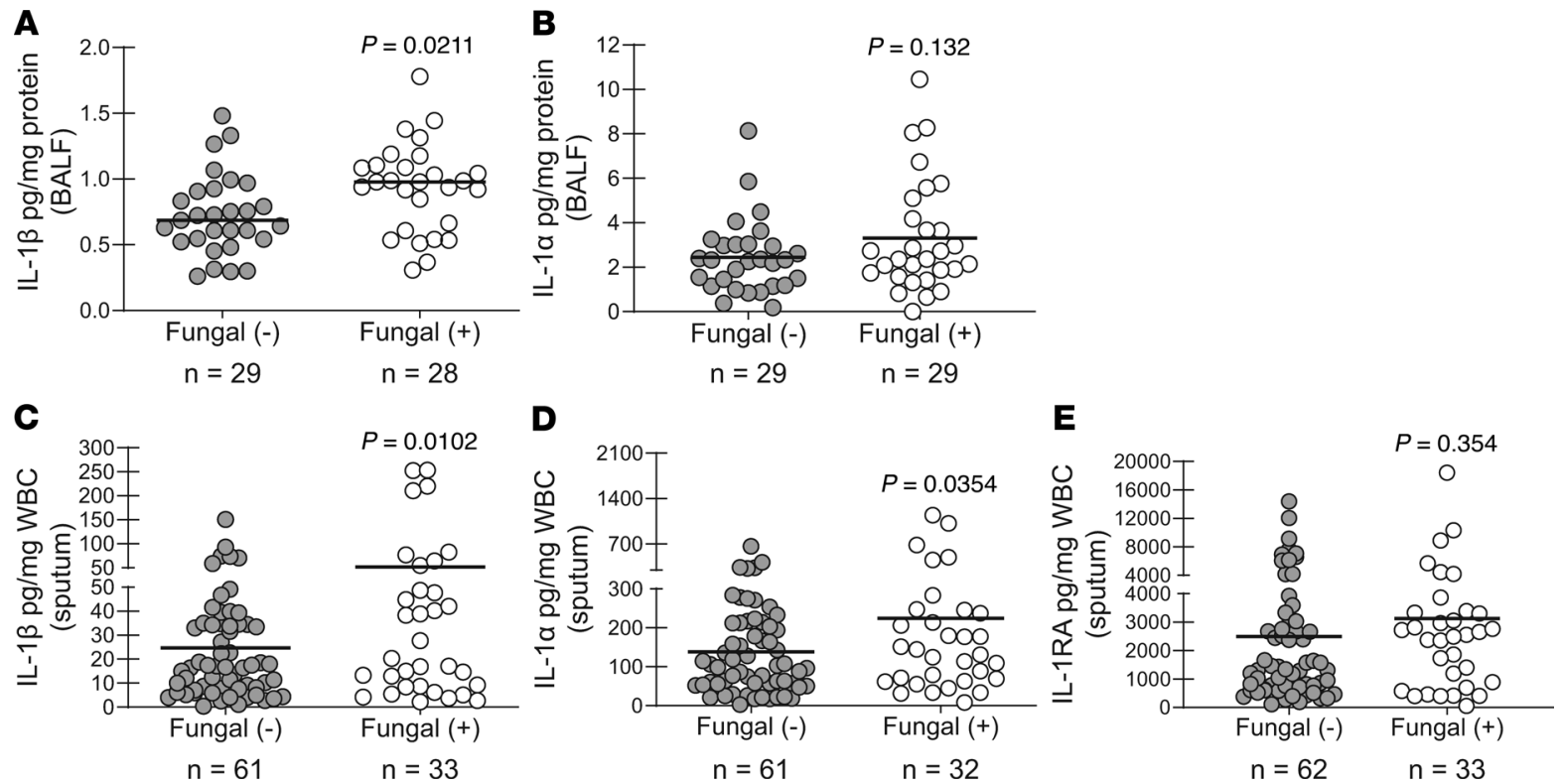

Figure 1. Human asthmatics sensitized to fungi have elevated IL-1 $\alpha$ and IL-1 $\beta$ levels in bronchoalveolar lavage fluid and sputum. Bronchoalveolar lavage fluid (BALF) or sputum was collected from subjects with atopic asthma who were sensitized (BALF, $n=28-29$; sputum, $n=32-33$ ) or were not sensitized (BALF, $n=29$; sputum, $n=61-62$ ) to fungi. IL-1 $\beta$ and IL-1 $\alpha$ were quantified in clarified (A and B) BALF and (C-E) sputum supernatants by MilliPlex. IL-1 $\beta$ data in BALF was previously reported in Table format (10). Data were normalized to the total protein content (BALF) or total WBC (sputum) of each sample and expressed as mean pg/mg protein or mean pg/total WBC (each symbol represents a single subject). $P$ values assessed using a 2-tailed Student's $t$ test.

status as non-SAFS asthmatics (7). Intriguingly, pediatric SAFS was associated with elevated levels of the type 2-associated cytokine IL-33 in bronchoalveolar lavage fluid (BALF). Likewise, a study of adult SAFS subjects also found elevated IL-33 in serum (6). IL-33 is a member of the IL-1 family of cytokines, which is composed of 11 members, 7 with proinflammatory characteristics (IL-1 $\alpha$, IL-1 $\beta$, IL-18, IL-33, IL-36 $\alpha$, IL-36 $\beta$, and IL-36 $\gamma$ ) and 4 with putative antiinflammatory characteristics (IL-1 receptor antagonist [IL-1RA], IL-36Ra, IL-37, and IL-38). We have previously shown, during experimental fungal-associated allergic airway inflammation, that the $\beta$-glucan receptor Dectin-1 (11), the proinflammatory cytokine IL-22 (11), and the common $\gamma$-chain cytokine IL-7 (10) contribute to disease severity, a component of which was the induction of the IL-1 family members IL- $1 \alpha$ and IL-1 $\beta$. Here, we observed higher levels of IL- $1 \alpha$, IL-1 $\beta$, and IL-1RA in lung samples from fungal-sensitized asthmatics compared with fungal-negative asthmatics, implicating a role for these factors in human fungal asthma pathogenesis. We therefore sought to clarify a role for these IL-1 family members in immunopathogenic responses during chronic exposure to A. fumigatus.

\section{Results}

Human asthmatics sensitized to fungi have elevated IL-1 $\alpha$ and IL-1 levels in BAL and sputum. We have recently reported that human asthmatics sensitized to fungi (National Heart Lung and Blood Institute's Severe Asthma Research Program [SARP] cohorts 1 and 2) have more severe disease as determined by multiple lung function (forced expiratory volume in 1 second, $\mathrm{FEV}_{1}$; concentration of methacholine producing 20\% drop in $\mathrm{FEV}_{1}, \mathrm{PC}_{20}$ ) and atopic measurements (serum IgE, blood eosinophils numbers) (10). In that report, Luminex-based analysis identified multiple cytokine, chemokine, and growth factors that were significantly elevated in BALF from fungal-sensitized asthmatics (10). Among these was IL-1RA, with regression analysis revealing that IL-1RA had a negative correlation with PC20 (i.e., higher IL-1RA levels correlated with lower PC20), which may suggest a role for IL-1RA in immunopathogenesis during fungal asthma (10). Alternatively, elevated IL-1RA levels could serve as a biomarker of lung function decline during fungal asthma. Here, we extend these observations by showing that IL-1 $\beta$ was also significantly higher in BALF from fungal-sensitized asthmatics (Figure 1A), whereas IL-1 $\alpha$ trended higher (Figure 1B). We also performed an additional analysis using the SARP 1 and 2 cohorts, using sputum from adult asthmatics who were atopic and sensitized to fungi vs. asthmatics who were atopic but not sensitized to fungi. Differences between fungal-sensitized vs. nonsensitized human asthmatics include current age and age of asthma onset, both of which were younger in fungal-sensitized asthmatics (Table 1). 
Table 1. Demographics and clinical characteristics of fungal (-) and fungal (+) asthmatics

\begin{tabular}{|c|c|c|c|}
\hline Variable & Fungal positive $(n=51)$ & Fungal negative $(n=77)$ & $P$ value \\
\hline Age (years) & $33 \pm 10.3$ & $39 \pm 13$ & 0.004 \\
\hline Asthma duration (years) & $21(17-29)$ & $22(11-32)$ & 0.511 \\
\hline Sex (\%female) & 60.80 & 71.40 & 0.288 \\
\hline $\begin{array}{l}\text { Race (\%nonhispanic } \\
\text { white/\%AA/\%other) }\end{array}$ & $45 / 51 / 4$ & $61 / 34 / 5$ & 0.194 \\
\hline Basal preBD FVC\%pred & $91.2 \pm 15.5$ & $87 \pm 15.6$ & 0.165 \\
\hline $\mathrm{FEV}_{1} / \mathrm{FVC}$ & $0.716(0.62-0.77)$ & $0.751(0.66-0.80)$ & $\underline{0.078}$ \\
\hline Max FEV $\%$ pred & $88.9 \pm 17.3$ & $89.2 \pm 15.8$ & 0.928 \\
\hline Max FVC\%pred & $100 \pm 14.4$ & $96 \pm 15.1$ & 0.124 \\
\hline Max reversal & $14.1(9-24)$ & $11.1(7.2-19.4)$ & 0.131 \\
\hline FeNO (ppb) & $26.7(17-60)$ & $27.3(18-43)$ & 0.761 \\
\hline Blood eos (count/ml) & $300(200-400)$ & $200(100-300)$ & 0.005 \\
\hline Blood eos (\%) & $5(3-7)$ & $3(1.5-4.5)$ & $<0.001$ \\
\hline Sputum neutrophil (\%) & $43(22-60)$ & $36(23-68)$ & 0.919 \\
\hline Sputum eosinophil (\%) & $1.5(0.2-5.9)$ & $0.6(0.2-2.1)$ & 0.101 \\
\hline Intubation for breathing (\%positive) & 23.90 & 9.40 & $\underline{0.07}$ \\
\hline $\begin{array}{l}\text { Hospitalization for breathing } \\
\text { (\%positive) }\end{array}$ & 42 & 32.50 & 0.367 \\
\hline Inhaled corticosteroid (\%positive) & 72.50 & 53.20 & 0.045 \\
\hline Long-acting $\beta$-agonist (\%positive) & 60.90 & 45.30 & 0.156 \\
\hline
\end{tabular}

AA, African American; FeNO, exhaled nitric oxide; preBD, prebronchodilator; FEV1\%pred; predicted percent of forced expiratory volume in 1 minute; eos, eosinophils. Underline and italics represent close to statistically significant; bold indicate statistically significant. Continuous variables (with numerical value) were tested by $t$ test if parametric (results presented as mean \pm SD), or Mann-Whitney $U$ (results presented as median [interquartile $25 \%-75 \%$ range]) if not meeting normality by Shapiro-Wilk test. Categorical variables (with yes or no response, e.g., intubation for breathing, or male or female) were tested by $\chi^{2}$ test or Fisher's Exact test (Sigmaplot v13.0).

There was also a trend toward lower $\mathrm{FEV}_{1}$ / forced vital capacity (FVC) (Table 1, $P=0.078$ ) in fungal-sensitized asthmatics, as we have recently reported (10). Fungal-sensitized asthmatics demonstrated significantly higher IgE levels, percentage and number of blood eosinophils, and usage of inhaled corticosteroids (Table 1). We observed significantly higher IL-1 $\beta$ (Figure 1C) and IL-1 $\alpha$ (Figure 1D) levels in sputum from fungal (+) asthmatics, or human asthmatics who are sensitized to fungal organisms. IL-1RA levels in sputum were not significantly different (Figure 1E). There was no difference in the ratio of IL-1RA to IL-1 $\alpha$ or IL-1 $\beta$ in either fungal-sensitized or nonsensitized asthmatics (data not shown). Overall, these results suggest that IL-1 signaling may exist as a central pathway in determining the severity of fungal asthma.

Signaling through the IL-1R1 worsens lung function during experimental fungal-associated allergic airway inflammation. As IL-1 $\alpha$ and IL-1 $\beta$ levels were elevated in fungal ( + ) asthmatics, we sought to determine the impact of IL-1 signaling on the severity of fungal asthma. Employing an experimental model of fungal-associated allergic airway inflammation $(10,11)$, mice deficient in the receptor for IL-1 $\alpha$ and IL-1 $\beta$ (Il1r1 ${ }^{-/-}$mice) demonstrated a dramatic decrease in central airway resistance (airway hyperreactivity; Figure 2A) and total lung resistance (Figure 2B) compared with WT control mice. H\&E staining of lung tissue sections revealed fewer inflammatory cells surrounding the airways and in alveolar spaces in $I l 1 r 1^{1 /-}$ mice (Figure 2C). Periodic acid-Schiff (PAS) staining of lung tissue sections demonstrated higher mucus staining in the airways of WT control mice that was much lower in $I l 1 r 1^{-1-}$ mice (Figure 2C). However, analysis of Muc5ac and Gob5 mRNA expression did not reveal any significant differences between WT and Illr1 $1^{-/-}$mice (Figure 2D). Thus, IL- $1 \alpha$ and IL-1 $\beta$ signaling during fungal-associated allergic airway inflammation contributes to lung inflammation, which negatively impacts lung function. 
A
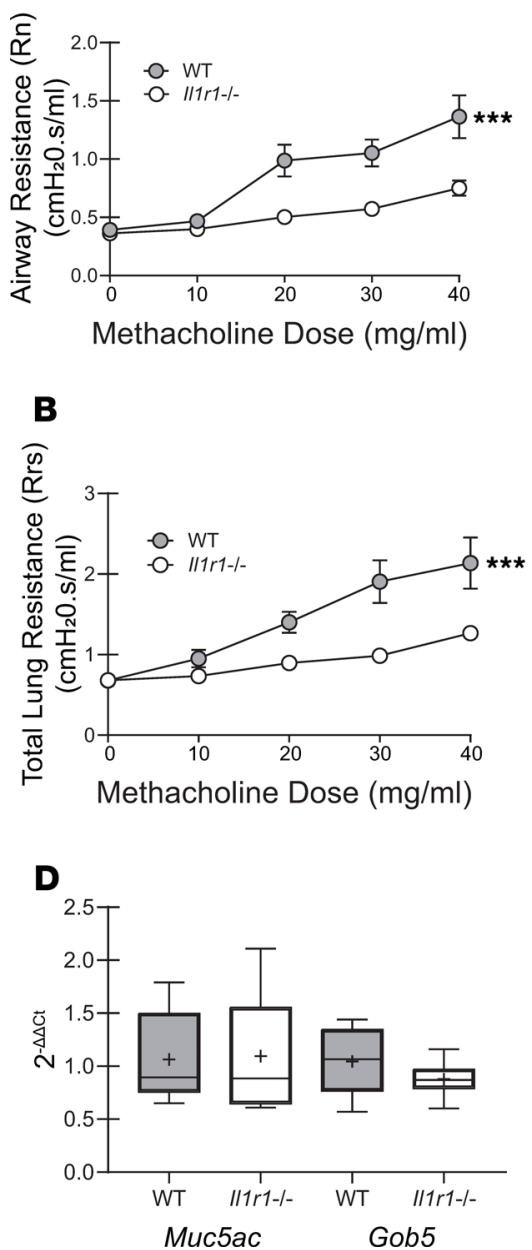

C

$H \& E$

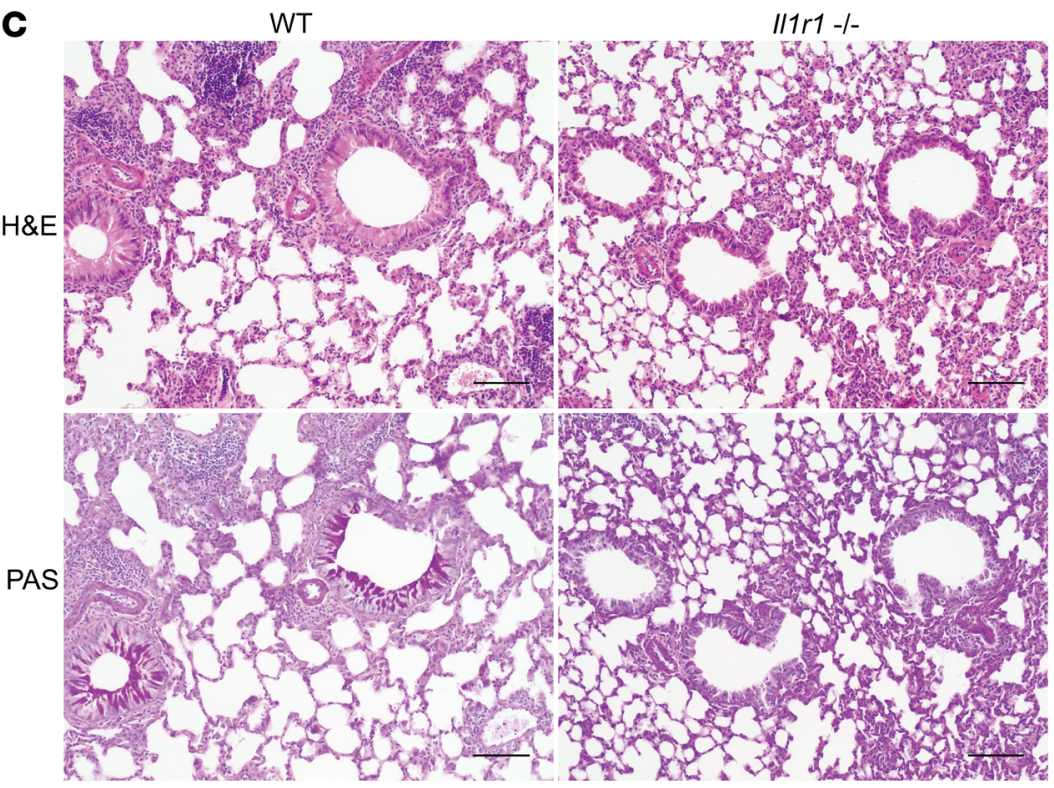

Figure 2. Signaling through the IL-1 receptor worsens lung function during experimental fungal-associated allergic airway inflammation. C57BL/6 and IL-1 receptor-deficient $\left(/ 17 \mathrm{r}^{-{ }^{--}}\right)$mice were chronically exposed to $A$. fumigatus as described in the Methods. Twenty-four hours after the last organism challenge, (A) airway (Newtonian) resistance and (B) total lung resistance was analyzed via mechanical ventilation using the flexiVent pulmonary function system. The figures illustrate cumulative data from 3 independent studies ( $n=4-5$ mice per group per study). Data expressed as mean \pm SEM. ${ }^{* *} P<0.001$ (2-way ANOVA). Representative (C) H\&E-stained (top images) and Periodic acid-Schiff-stained (PASstained; bottom images) lung sections from WT (left images) and $1 / 1 r 1^{-1-}$ (right images) mice. Original magnification, 20x. Scale bar: $100 \mu \mathrm{m}$. (D) Twenty-four hours after the last challenge, the left lungs were collected, and Muc5ac and Gob5 gene expression was quantified by real-time PCR and normalized to HPRT. Gene expression as $2^{-\Delta \Delta c t}$. The figure illustrates cumulative data from 2 independent studies ( $n=4$ mice per group per study). Quantitative data is represented as a box-and-whisker plot, with bounds ranging from 25 th to 75 th percentile, the line representing the median, whiskers ranging from minimum to maximum values, and + indicating the mean.

$I L-1 R A$ regulates the severity of experimental fungal-associated allergic airway inflammation. As mentioned previously, we recently reported that IL-1RA was elevated in BALF from fungal (+) asthmatics and negatively correlated with lung function (10). Since these results suggested that high levels of IL-1RA may act in an immunopathogenic manner during fungal asthma, we sought to clarify the role of IL-1RA. In contrast to mice deficient in the IL-1 receptor (IL-1R1), mice deficient in the IL-1RA ( $I l 1 \mathrm{rn}^{-1-}$ mice) subjected to fungal-associated allergic airway inflammation demonstrated a profound decrement in lung function, as evidenced by a significant increase in central airway resistance (Figure 3A) and total lung resistance (Figure 3B). H\&E staining and PAS staining (Figure 3C) of lung tissue sections again demonstrated a low to moderate level of inflammatory cell recruitment and robust mucus staining in WT control mice. In direct contrast, $I l 1 r^{-/-}$mice surprisingly did not demonstrate mucus staining — rather, there was evidence for a destructive inflammatory response leading to either the collapse or occlusion of airways, as well as consolidation of the alveolar space. Based on these observations, we examined additional lung function measurements that reflect peripheral airway and parenchymal lung function. This analysis demonstrated that dynamic compliance, a measure of the ease with which the respiratory system can be extended, was significantly lower in $111 \mathrm{rn}^{-1}$ mice (Figure 3D). In addition, tissue damping, a measure of resistance to air flow in the peripheral airways (Figure 3E), and tissue elastance, a measure of tissue stiffness (Figure 3F), were increased in $111 \mathrm{rn}^{-1-}$ mice. Despite these changes in lung function, analysis of $M u c 5 a c$ and Gob5 mRNA expression demonstrated significantly lower levels in $\mathrm{Illm}^{-/-}$mice (Figure 3G). Thus, IL-1RA is required for regulating lung responses that contribute to lung function impairment during fungal-associated allergic airway inflammation.

Neutrophils and their associated inflammatory mediators are differentially regulated by IL-1RI and IL-1RA during experimental fungal-associated allergic airway inflammation. Continued phenotyping of asthma subsets has led to the classification of allergic asthmatics into 2 broad inflammatory categories, type 2, 
A
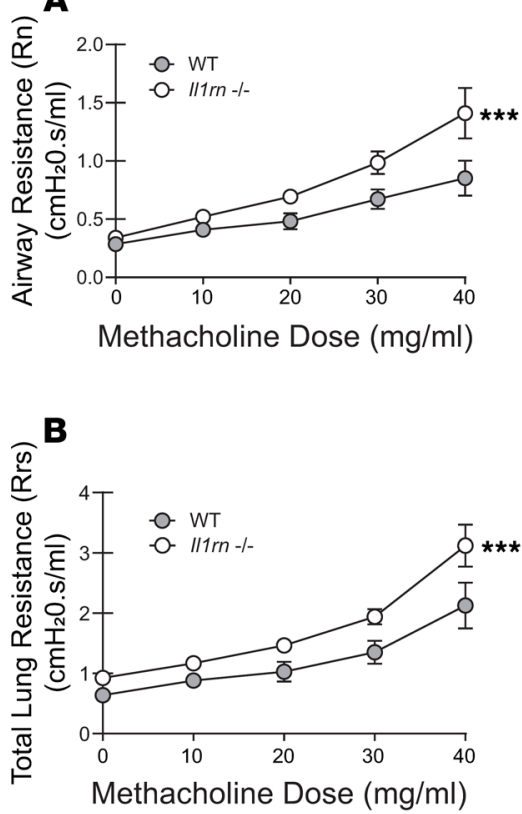

D

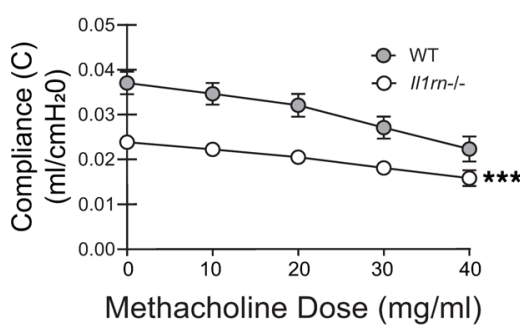

G

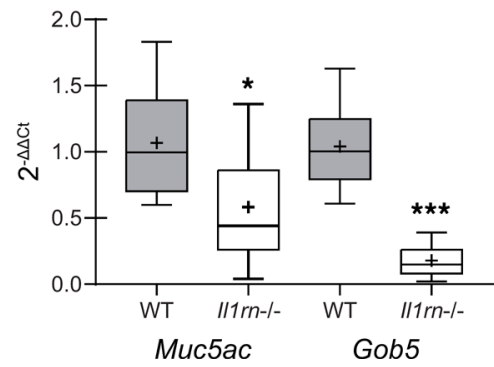

C

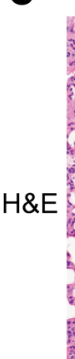

WT

/11rn -/-

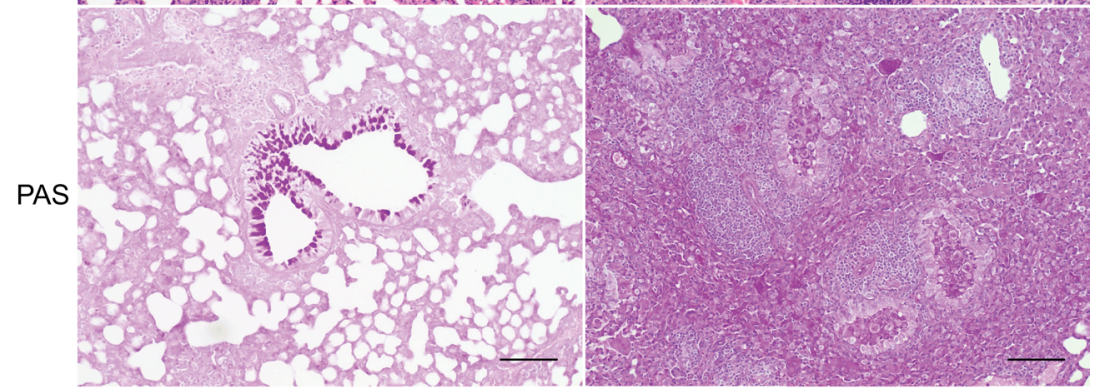

E
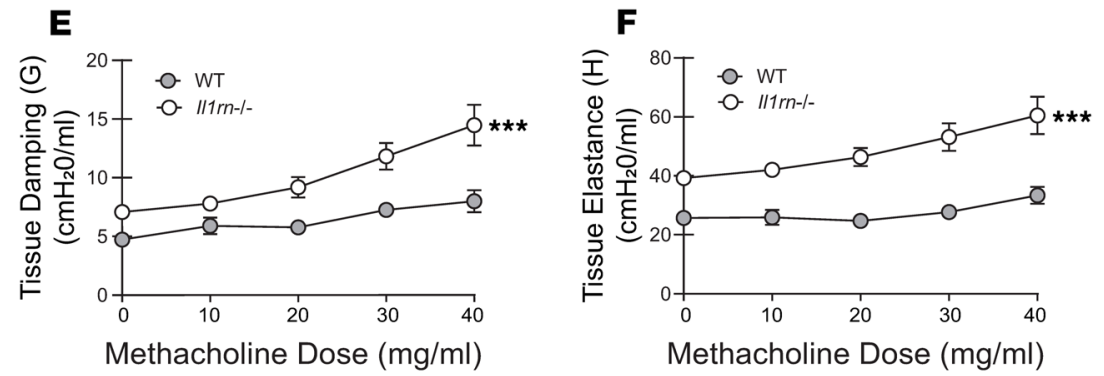

Figure 3. IL-1 receptor antagonist regulates the severity of experimental fungal-associated allergic airway inflammation. C57BL/6 WT and IL-1 receptor antagonist-deficient $\left(/ 11 \mathrm{rn}^{-1}\right)$ mice were chronically exposed to $A$. fumigatus as described in the Methods. Twenty-four hours after the last organism challenge, (A) airway (Newtonian) resistance and (B) total lung resistance was analyzed via mechanical ventilation using the flexiVent pulmonary function system. The figures illustrate cumulative data from 2 independent studies ( $n=4-5$ mice per group per study). Data are expressed as mean \pm SEM. ${ }^{* * *} P<0.001$ ( 2 -way ANOVA). Representative (C) H\&E- and PAS-stained lung sections from WT (left images) and I/1rn $\mathrm{r}^{-1-}$ (right images) mice. Original magnification, 20x. Scale bar: $100 \mu \mathrm{m}$. (D) Compliance, (E) tissue damping, and (F) tissue elastance was analyzed via mechanical ventilation using the flexiVent pulmonary function system. The figures illustrate cumulative data from 2 independent studies ( $n=4-5$ mice per group per study). Data expressed as mean \pm SEM. ${ }^{* * *} P<0.001$ ( 2 -way ANOVA). (C) Twenty-four hours after the last challenge, the left lungs were collected, and Muc5ac and Gob5 gene expression was quantified by real-time PCR and normalized to HPRT. Gene expression presented as $2^{-\Delta \Delta C t}$. The figure illustrates cumulative data from 2 independent studies ( $n=4$ mice per group per study). Quantitative data is represented as a box-and-whisker plot, with bounds ranging from 25th to 75th percentile, the line representing the median, whiskers ranging from minimum to maximum values, and + indicating the mean.

eosinophilic-dominated asthma; and type 17, neutrophilic-dominated asthma (12), the latter of which is often viewed as more severe and steroid resistant. However, neutrophils are central effector cells against fungi - A. fumigatus, in particular (13); thus, they are an expected component of the immune response to inhaled fungi. We have previously reported that neutrophil levels in experimental fungal-associated 
A

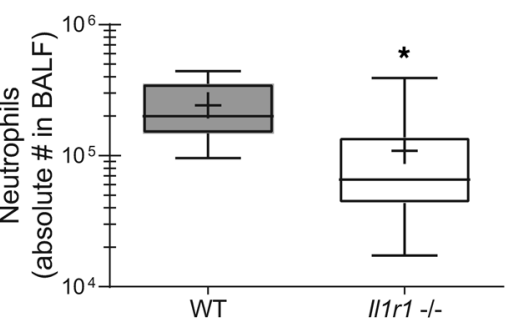

D

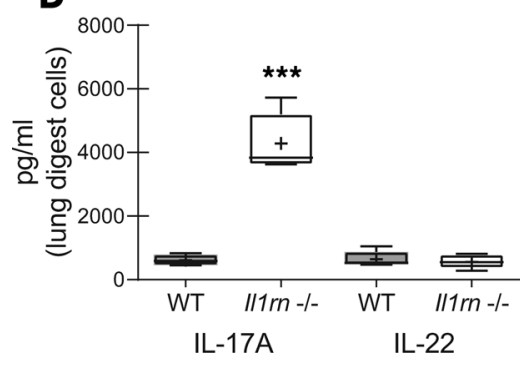

B

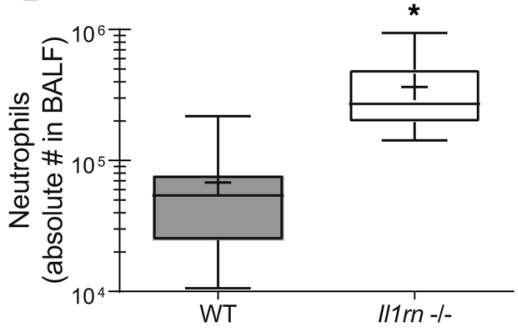

E

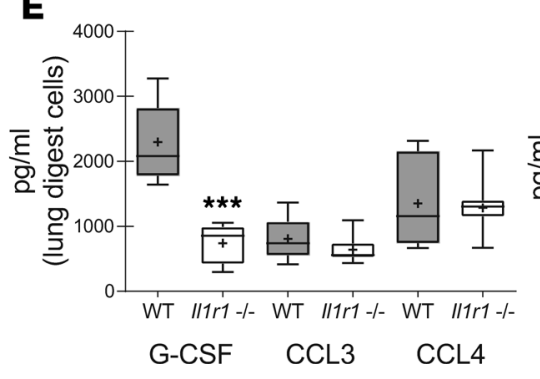

C

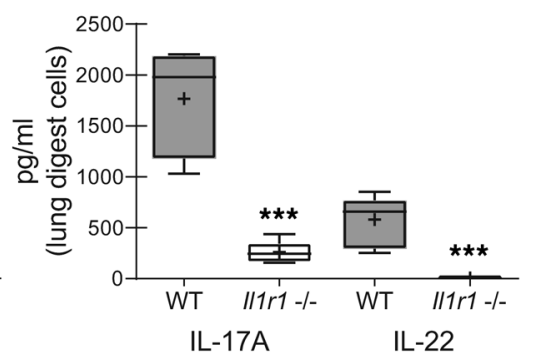

$\mathbf{F}$

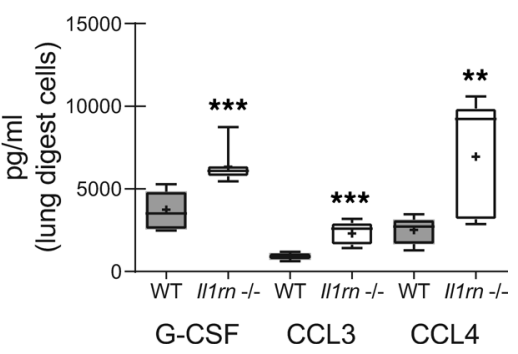

Figure 4. Neutrophils and their associated inflammatory mediators are differentially regulated by IL-1R1 and IL-1RA during experimental fungalassociated allergic airway inflammation. C57BL/6 WT, IL-1 receptor-deficient (/11r $\mathrm{r}^{-/-}$), and IL-1 receptor antagonist-deficient (I/1rn $\left.{ }^{-/-}\right)$mice were chronically exposed to A. fumigatus as described in the Methods. (A and B) Twenty-four hours after the last challenge, lung cells from (A) WT and $1 / 1 \mathrm{r}^{-1-}$ mice and from (B) WT and $1 / 1 \mathrm{rn}^{-1-}$ mice were isolated by bronchoalveolar lavage, enumerated, Fc-blocked, stained with a live/dead staining kit, and stained for neutrophils ( $\mathrm{CD}_{45}+, \mathrm{CD}_{11} \mathrm{~b}^{+}, \mathrm{Ly}-6 \mathrm{C}^{+}$, Siglec $\mathrm{F}^{-}$). The figures illustrate cumulative data from 2-3 independent studies $(n=4-5$ mice per group per study). (C and D) Twenty-four hours after the last challenge, right lungs were collected and enzymatically digested, and unfractionated lung cells were cocultured with $A$. fumigatus conidia for 24 hours at a cell/organism ratio of 1:1. IL-17A and IL-22 levels in lung digest cell culture supernatants from (C) WT and $/ 11 \mathrm{r}^{-1-}$ mice and (D) WT and $/ 11 \mathrm{rn} \mathrm{n}^{-/-}$mice were quantified by MilliPlex or ELISA. The figures illustrate cumulative data from 2 independent studies ( $n=3$ mice per group per study). (E and F) Samples obtained as described for $\mathbf{C}$ and $\mathbf{D}$. G-CSF, CCL3, and CCL4 levels in lung digest cell culture supernatants from (E) WT and $/ / 1 r 1^{-1-}$ mice and (F) WT and $/ / 1 r n^{-1-}$ mice were quantified by MilliPlex. The figures illustrate cumulative data from 3 independent studies ( $n=3-5$ mice per group per study). For all graphs, quantitative data are represented as a box-and-whisker plot, with bounds ranging from 25 th to 75 th percentile, the line representing the median, whiskers ranging from minimum to maximum values, and + indicating the mean. ${ }^{*} P<0.05,{ }^{*} P<0.01$, and ${ }^{* *} P<0.001$ (2-tailed Student's $t$ test).

allergic airway inflammation may be associated with a lower inflammatory profile and improved lung function (11), although in some cases, experimental fungal-associated allergic airway inflammation may be severe in the absence of changes in the neutrophil population (10). Here, the absence of IL-1R1 signaling was associated with decreased neutrophil recruitment during experimental fungal-associated allergic airway inflammation, as $1 l 1 \mathrm{rl}^{-1-}$ mice had a $50 \%$ reduction in neutrophil recruitment after chronic fungal exposure (Figure 4A). In contrast, IL-1RA was required to regulate the magnitude of neutrophil recruitment, as $I l 1 \mathrm{rn}^{-/}$mice demonstrated a 3 -fold increase in neutrophil numbers during chronic fungal exposure (Figure 4B). Representative flow plots are included in Supplemental Figure 1 (supplemental material available online with this article; https://doi.org/10.1172/jci.insight.129055DS1). The magnitude of neutrophil recruitment in these mice was directly associated with the level of IL-17A, with significantly lower levels produced by lung digest cells from mice deficient in IL-1R1 signaling (Figure 4C) and significantly higher levels produced in the absence of IL-1RA (Figure 4D). In contrast, while IL-22 production was dependent on the IL-1R1 (Figure 4C), IL-1RA was not required for IL-22 regulation (Figure 4D). Additional analyses showed that the proneutrophil survival factor granulocyte CSF (G-CSF) was dependent, at least in part, on IL-1R1 signaling (Figure 4E), and IL-1RA was required for maintaining G-CSF at WT levels during chronic fungal exposure (Figure 4F). In contrast, the neutrophil chemoattractants CCL3 and CCL4 were not dependent on IL-1R1 signaling (Figure 4E) but were regulated by IL-1RA (Figure 4F). We also observed decreased CXCL1 levels in $I l 1 r 1^{-1-}$ mice, although this was not regulated by IL-1RA (i.e., $I l 1 \mathrm{rn}^{-/-}$mice did not demonstrate an increase in CXCL1; data not shown). Thus, the severity of experimental fungal-associated allergic airway inflammation is associated with neutrophil levels in the lung, where IL-1R1 signaling is required for the induction of proneutrophil survival factors and IL-1RA is required for controlling the level of these survival factors, as well as neutrophil chemoattractants. 
A

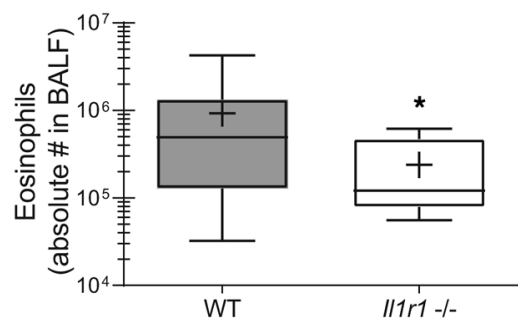

D

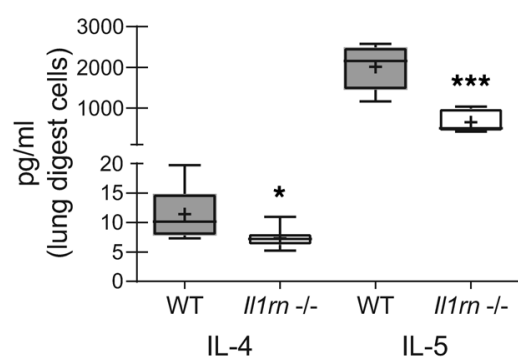

G

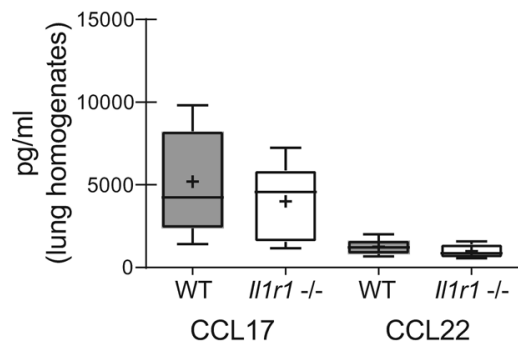

J

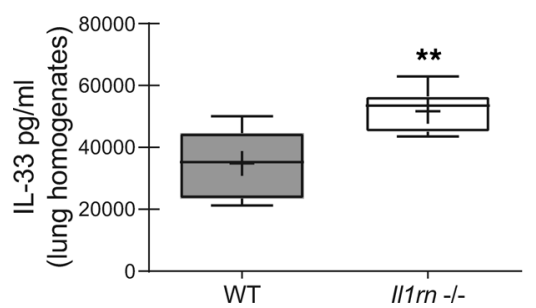

B

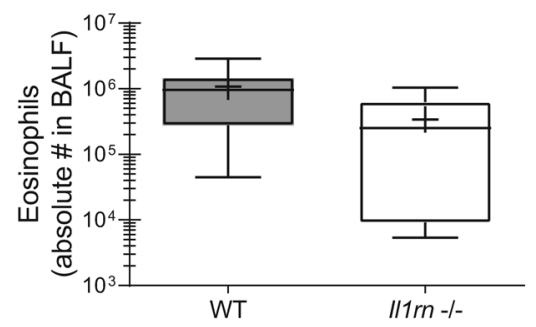

E

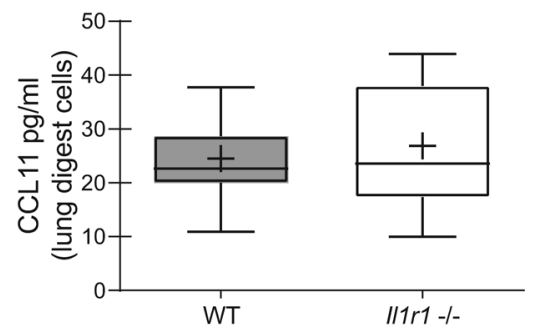

H

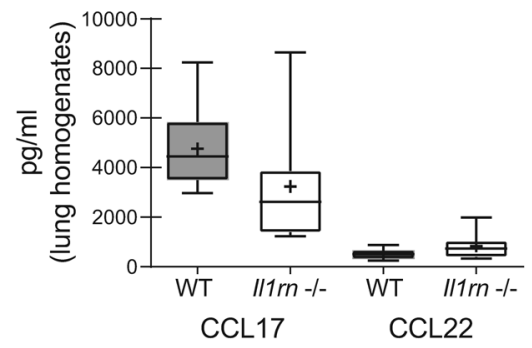

C

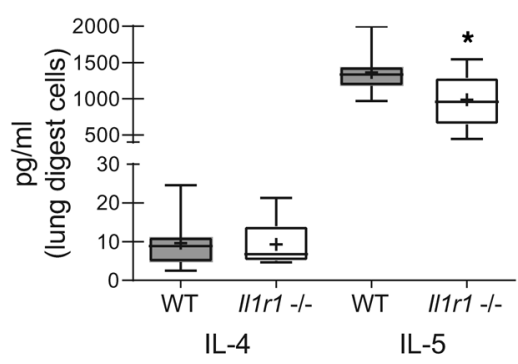

F

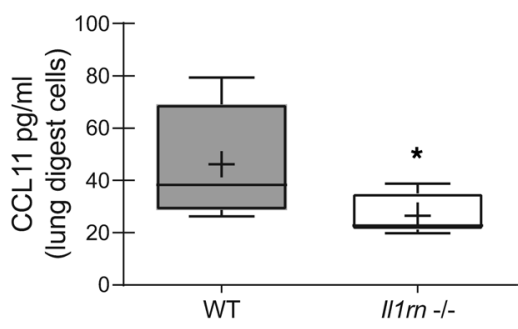

I

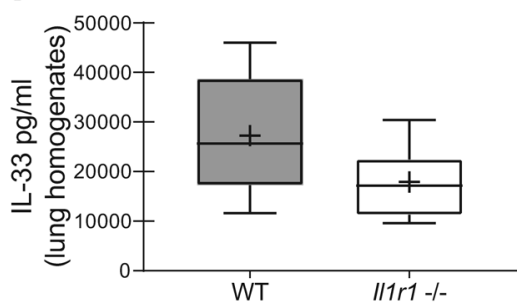

Figure 5. The absence of IL-1R1 and IL-1RA results in varied type 2 responses during experimental fungal-associated allergic airway inflammation.

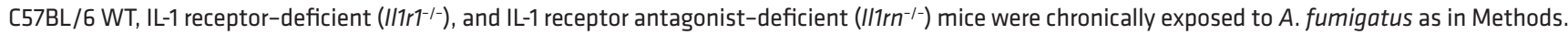
(A and B) Twenty-four hours after the last challenge, lung cells from (A) WT and I/1r $\mathrm{r}^{-/-}$mice and (B) WT and $/ 11 \mathrm{rn}^{-1-}$ mice were isolated by bronchoalveolar lavage, enumerated, Fc-blocked, stained with a live/dead staining kit, and stained for eosinophils (CD45+, CD11b+, Siglec $\mathrm{F}^{+}$, Ly- $6 \mathrm{C}^{-}$). The figures illustrate cumulative data from 2-4 independent studies ( $n=4-5$ mice per group per study). (C-F) Twenty-four after last challenge, right lungs were collected and enzymatically digested, and unfractionated lung cells were cocultured with $A$. fumigatus conidia for 24 hours at a cell/organism ratio of 1:1. IL-4, IL-5, and CCL11 levels in lung digest cell culture supernatants from (C and E) WT and $/ 11 r^{-1-}$ mice and from ( $\mathbf{D}$ and $\mathbf{F}$ ) WT and $/ 17 r n^{-1-}$ mice were quantified by MilliPlex. The figures illustrate cumulative data from 3-4 independent studies ( $n=3-5$ mice per group per study). (G-J) Twenty four hours after last challenge, left lungs were collected and homogenized, and CCL17, CCL22, and IL-33 levels were quantified by ELISA in clarified lung homogenates from (G and I) WT and $\| 11 \mathrm{r}^{-1-}$ mice and from ( $\mathbf{H}$ and $\left.\mathbf{J}\right) \mathrm{WT}$ and $I / 1 \mathrm{r} \mathrm{n}^{-1-}$ mice. The figures illustrate cumulative data from 2-3 independent studies ( $n=3-5$ mice per group per study) For all graphs, quantitative data are represented as a box-and-whisker plot, with bounds ranging from 25 th to 75 th percentile, the line representing the median, whiskers ranging from minimum to maximum values, and + indicating the mean. ${ }^{*} P<0.05,{ }^{* *} P<0.01$, and ${ }^{* * *} P<0.001$ ( 2 -tailed Student's $t$ test).

The absence of IL-1R1 and IL-1RA results in varied type 2 responses during experimental fungal-associated allergic airway inflammation. The dominant immune response in allergic asthma immunopathogenesis is often considered to be type 2 and eosinophil centric. Similar to our findings with neutrophils detailed above, experimental fungal-associated allergic airway inflammation severity may (10) or may not (11) be associated with the level of eosinophils. IL-1R1 deficiency resulted in a significant reduction in the number of eosinophils recruited 
A

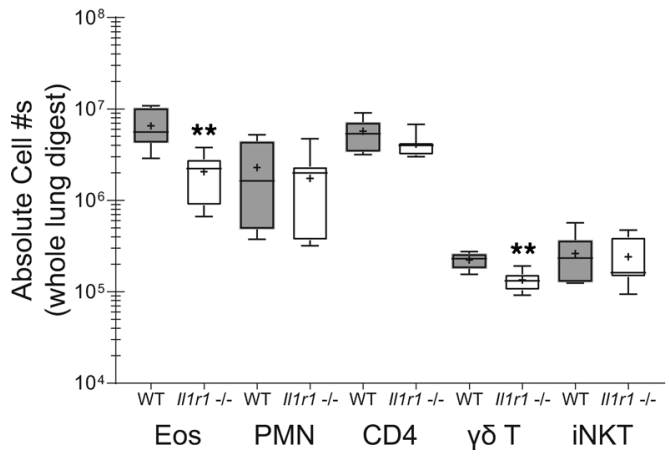

B

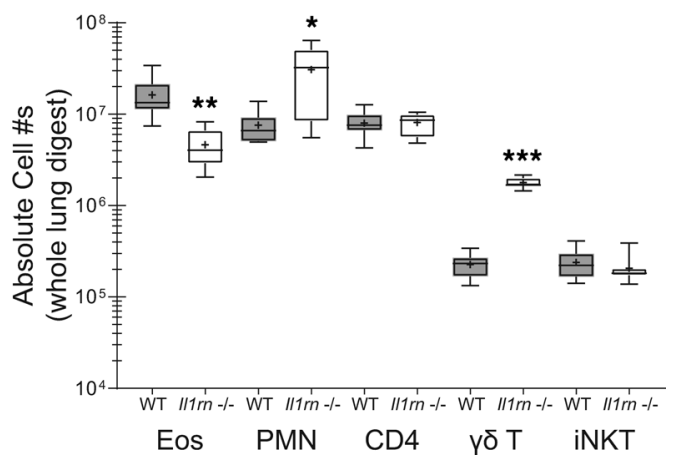

Figure 6. Cellular changes in whole lung during experimental fungal-associated allergic airway inflammation.

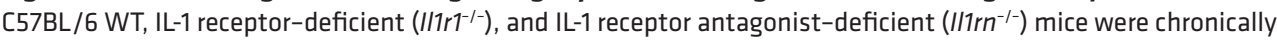
exposed to $A$. fumigatus as in Methods. Twenty-four hours after the last challenge, right lungs were collected, enzymatically digested, Fc-blocked, stained with a live/dead staining kit, and stained for eosinophils (CD45+, CD11b+, Siglec

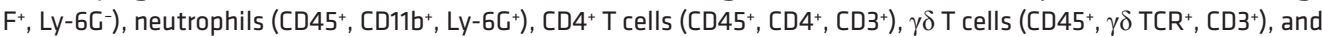
iNKT cells (TCR $\beta^{+}$, PBS-57 CD1d tetramer ${ }^{+}$) in (A) WT and $111 r^{-1-}$ mice and (B) WT and $/ 11 r^{-1-}$ mice. The figures illustrate cumulative data from 2 independent studies ( $n=3-4$ mice per group per study). For all graphs, quantitative data are represented as a box-and-whisker plot, with bounds ranging from 25 th to 75 th percentile, the line representing the median, whiskers ranging from minimum to maximum values, and + indicating the mean. ${ }^{*} P<0.05,{ }^{* *} P<0.01$, and ${ }^{* * *} P<0.001$ (2-tailed Student's $t$ test).

to the lung during experimental fungal-associated allergic airway inflammation (Figure 5A). Somewhat surprisingly, IL-1RA deficiency also resulted in a trend toward lower eosinophil numbers $(P=0.089$; Figure 5B) Representative flow plots are included in Supplemental Figure 1. Regarding type 2 responses, IL-4 was not different between Ill1r1 ${ }^{-/-}$mice and WT controls, whereas IL-5 was significantly lower (Figure 5C). In contrast, both IL-4 and IL-5 were significantly lower in $I l 1 r^{-1-}$ mice (Figure 5D). IL-13 was not consistently produced during fungal-associated allergic airway inflammation, whereas IL-9 levels were not different between WT and $I l 1 r^{-/-}$mice or WT and Illrn ${ }^{-/-}$mice (data not shown). The major eosinophil chemoattractant CCL11 was not modulated by IL-1R1 deficiency (Figure 5E) but was significantly lower in the absence of IL-1RA (Figure 5F). The proallergic chemokines CCL17 and CCL22, which we have previously employed as biomarkers of fungal-associated allergic airway inflammation severity $(10,11)$ and are recognized biomarkers in human allergic bronchopulmonary aspergillosis $(14,15)$, were not affected by IL-1R1 deficiency (Figure 5G) or IL-1RA deficiency (Figure 5H). Finally, deficiency in IL-1R1 signaling did not impact the levels of the IL-1 family and pro-type 2 cytokine IL-33 (Figure 5I), while IL-33 was increased in the absence of IL-1RA (Figure $5 \mathrm{~J})$. Thus, analogous to neutrophils, the severity of experimental fungal-associated allergic airway inflammation in IL-1R1-deficient mice is associated with eosinophil levels and proeosinophil survival factors. In contrast, severity of experimental fungal-associated allergic airway inflammation in IL-1RA-deficient mice did not involve heightened type 2 or eosinophil-centric responses.

Cellular changes in whole lung during experimental fungal-associated allergic airway inflammation. As Ill $\mathrm{rn}^{-1}$ mice had histological and physiological evidence of peripheral airway and parenchymal lung dysfunction (Figure 3), we examined cellular changes in the whole lung to complement measurements made in the airway (assessed in lung lavage fluid; Figure 4 and Figure 5). For this, we performed flow cytometry on cells collected from enzymatic digests of whole lungs. This analysis demonstrated that eosinophils, but not neutrophils, were significantly decreased in whole lungs from $I l 1 \mathrm{rl}^{-1-}$ mice (Figure 6A), whereas both were decreased when assessed in lung lavage fluid (Figure 4A and Figure 5A). Regarding $\mathrm{Ill} \mathrm{rn}^{-1-}$ mice, eosinophils were significantly decreased and neutrophils were significantly increased in whole lungs (Figure 6B), which is consistent with the lung lavage fluid data (Figures $4 \mathrm{~B}$ and Figure $5 \mathrm{~B}$ ). We have previously reported that IL-17A and IL-22 function in an immunopathogenic manner during experimental fungal-associated allergic airway inflammation $(11,16)$. We recently reported that $\mathrm{CD} 4^{+} \mathrm{T}$ cells, as well as the innate lymphocytes $\gamma \delta \mathrm{T}$ cells, and invariant NKT cells (iNKT) cells were cellular sources of IL-22 (and likely IL-17A) in the lung during experimental fungal-associated allergic airway inflammation (10). As IL-17A and IL-22 production by lung cells was different between $I l 1 \mathrm{rr}^{-/-}$and $I l 1 \mathrm{rn}^{-/-}$mice (Figure 4), we determined the levels of $\mathrm{CD}^{+}{ }^{+} \mathrm{T}$ cells, $\gamma \delta \mathrm{T}$ cells, and iNKT cells in whole lungs. Results showed that 
A

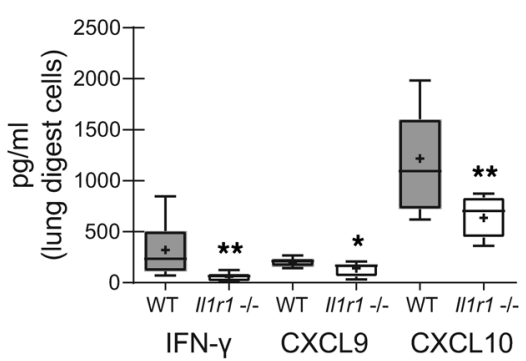

B

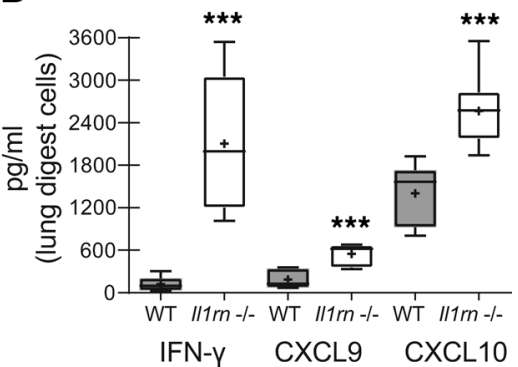

Figure 7. Type 1 responses are differentially regulated by IL-1R 1 and IL-1RA during experimental fungal-associated allergic airway inflammation. C57BL/6 WT, IL-1 receptor-deficient $\left(/ 11 r^{-1-}\right)$, and IL-1 receptor antagonist-deficient $\left(I / 1 \mathrm{rn}^{-/-}\right)$mice were chronically exposed to A. fumigatus as in Methods. (A and B) Twenty-four hours after last challenge, right lungs were collected and enzymatically digested, and unfractionated lung cells were cocultured with $A$. fumigatus conidia for 24 hours at a cell/organism ratio of 1:1. IFN- $\gamma$, CXCL9, and CXCL10 levels in lung digest cell culture supernatants from (A) WT and $/ 11 \mathrm{r}^{-1-}$ mice and from (B) WT and $/ 11 \mathrm{rn} \mathrm{r}^{-1-}$ mice were quantified by MilliPlex. The figures illustrate cumulative data from 3 independent studies (3-5 mice per group per time point). For all graphs, quantitative data are represented as a box-and-whisker plot, with bounds ranging from 25th to 75th percentile, the line representing the median, whiskers ranging from minimum to maximum values, and + indicating the mean. ${ }^{*} P<0.05,{ }^{* *} P<0.01$, and ${ }^{* * *} P<0.001$ ( 2 -tailed Student's $t$ test).

$\mathrm{CD}^{+} \mathrm{T}$ cells and iNKT cells were not different between WT and $I l 1 r 1^{1^{\prime-}}$ mice; however, there was a significant decrease in $\gamma \delta \mathrm{T}$ cells (Figure 6A). The decrease in $\gamma \delta \mathrm{T}$ cells was associated with lower IL-17A

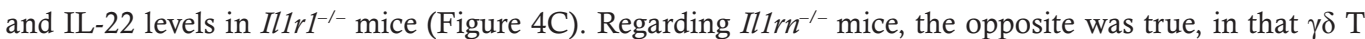
cells were significantly increased, but there were no changes in the levels of CD4 ${ }^{+} \mathrm{T}$ cells and iNKT cells (Figure 6B). The increase in $\gamma \delta \mathrm{T}$ cells was associated with higher IL-17A levels in Illrn ${ }^{-1-}$ mice (Figure 4D). Representative flow plots are included in Supplemental Figure 2. Thus, analysis of cell populations in whole lung generally replicates the findings in lung lavage fluid and is associated with aspects of the lung immune response during experimental fungal-associated allergic airway inflammation.

Type 1 responses are differentially regulated by $I L-1 R 1$ and $I L-1 R A$ during experimental fungal-associated allergic airway inflammation. Recent studies have implicated a role for IFN- $\gamma$-associated type 1 responses in severe asthma pathogenesis and corticosteroid insensitivity $(17,18)$. Although some data exit for IL-1R1-mediated regulation of type I IFNs (IFN- $\alpha$, IFN- $\beta$ ) (19), it is not clear if IL- $1 \alpha$ and/or IL- $1 \beta$ regulate type II IFNs (IFN- $\gamma$ ). However, after chronic exposure to A. fumigatus, production of IFN- $\gamma$ by lung digest cells demonstrated a profound requirement for IL-1R1 signaling (Figure 7A). Similarly, the IFN- $\gamma$ inducible chemokines CXCL9 and CXCL10 showed significantly impaired induction in $1 / 1 \mathrm{r}^{-/-}$mice compared with WT controls (Figure 7A). IL-1-mediated induction of IFN- $\gamma$ was tightly controlled, as the absence of IL-1RA resulted in more than a 10 -fold increase in IFN- $\gamma$ production (Figure 7B). Similarly, this lack of IFN- $\gamma$ regulation resulted in enhanced CXCL9 and CXCL10 production (Figure 7B). Thus, IL-1R1 signaling drives IFN- $\gamma$-mediated responses during experimental fungal-associated allergic airway inflammation, while IL-1RA functions as a critical regulator of these responses.

In vivo administration of human IL-1RA improves lung function during experimental fungal-associated allergic airway inflammation. Human recombinant IL-1RA is used to treat many autoimmune and inflammatory diseases in which IL-1-induced responses are associated with immunopathology and poor outcomes (20, 21). As our results implicate IL-1R1 signaling as a major immunopathogenic pathway in fungal asthma and describe IL-1RA as an important regulator of this pathway, we assessed the potential impact of human recombinant IL-1RA (Kineret/anakinra) on AHR. Administration of Kineret/anakinra throughout the development of experimental fungal-associated allergic airway inflammation had a modest effect on central airway resistance at a dose of $10 \mathrm{mg} / \mathrm{kg}$ (which is 10-fold less than the recommended dose in humans) (Figure 8A). However, Kineret/anakinra at this dose demonstrated more potent efficacy in reducing dynamic lung resistance compared with vehicle-treated mice (Figure $8 \mathrm{~B}$ ). A more profound effect on central airway resistance was observed at a dose of $50 \mathrm{mg} / \mathrm{kg}$ (Figure 8C), although this dose did not further improve total lung resistance (Figure 8D). Improved lung function was associated with significantly lower production of the type 1-associated cytokine IFN- $\gamma$ and associated chemokines CXCL9 and CXCL10 (Figure 8E) and with lower production of the type 17 associated cytokine IL-17A, but not IL-22 (Figure 8F), in Kineret/anakinra mice. There was no effect of Kineret/anakinra 
A

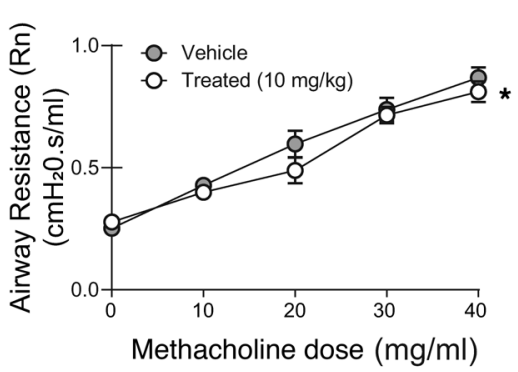

D

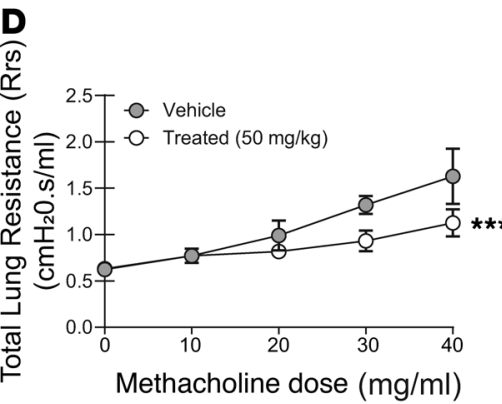

G

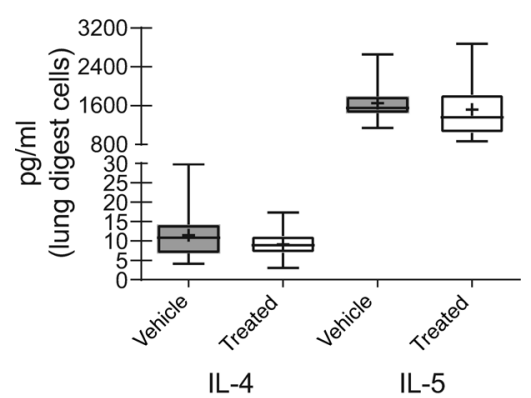

B

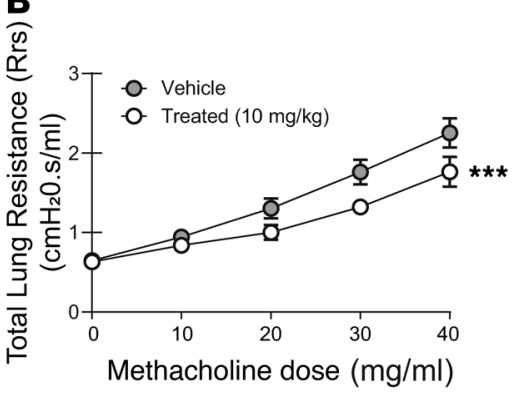

E

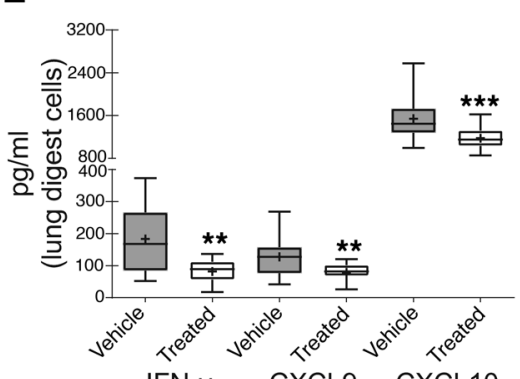

H

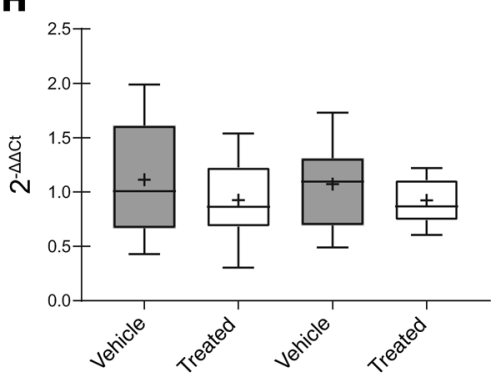

C

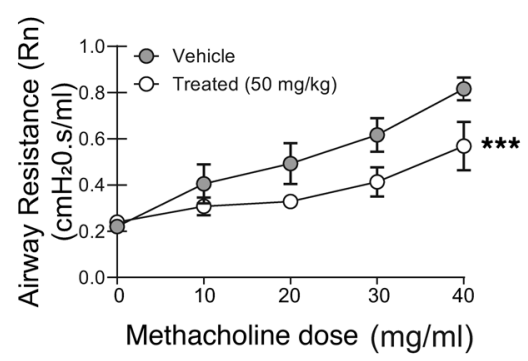

F

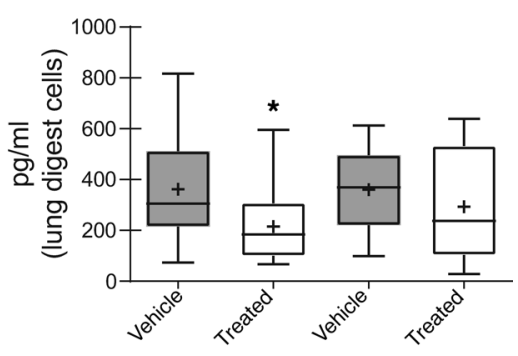

IL-17A

IL-22

Gob5

Figure 8. In vivo administration of human IL-1RA improves lung function during experimental fungal-associated allergic airway inflammation. C57BL/6 WT mice were chronically exposed to A. fumigatus as described in Methods and treated daily from days 0-16 with $10 \mathrm{mg} / \mathrm{kg}$ or $50 \mathrm{mg} / \mathrm{kg}$ human recombinant IL-1RA (Kineret/anakinra) or vehicle i.p. Twenty-four hours after the last organism challenge, (A and C) Airway (Newtonian) resistance and (B and $\mathbf{D}$ ) total lung resistance was analyzed via mechanical ventilation using the flexiVent pulmonary function system. The figures illustrate cumulative data from 2 independent studies ( $n=4-5$ mice per group per study). Data expressed as mean \pm SEM. ${ }^{*} P<0.05$ and ${ }^{* * *} P<0.001$ (2-way ANOVA). (E-G) Twenty-four hours after the last organism challenge, the right lungs were collected and enzymatically digested, and unfractionated lung cells were cultured for 24 hours in the presence of $A$. fumigatus conidia at a cell/organism ratio of 1:1. (E) IFN- $\gamma$, CXCL9 and CXCL10 levels, (F) IL-17A and IL-22 levels, and (C) IL-4 and IL-5 levels in lung digest cell culture supernatants from vehicle treated vs. Kineret/anakinra were quantified by MilliPlex or ELISA; Milliplex was employed for all cytokines except IL-22, and ELISA was used for IL-22. The figures illustrate cumulative data from 2 independent studies ( $n=4-5$ mice per group per study). Data are represented as a box-and-whisker plot, with bounds ranging from 25th to 75th percentile, the line representing the median, whiskers ranging from minimum to maximum values, and + indicating the mean. ${ }^{*} P<0.05,{ }^{*} P<0.01$, and ${ }^{* * *} P<0.001$ ( 2 -tailed Student's $t$ test). (H) Twenty-four hours after the last challenge, the left lungs were collected, and Muc5ac and Gob5 gene expression was quantified by real-time PCR and normalized to HPRT. Gene expression presented as $2^{-\Delta \Delta c t}$. The figure illustrates cumulative data from 2 independent studies ( $n=4$ mice per group per study). Data are represented as a box-and-whisker plot, with bounds ranging from 25th to 75th percentile, the line representing the median, whiskers ranging from minimum to maximum values, and + indicating the mean. ${ }^{*} P<0.05,{ }^{* *} P<0.01$, and ${ }^{* * *} P<0.001$ (2-tailed Student's $t$ test).

treatment on the production of the type 2 cytokines IL-4 and IL-5 (Figure 8G). There was also no difference in Muc5ac and Gob5 mRNA expression between vehicle and Kineret/anakinra-treated mice (Figure 8H) and no significant differences in any cell population in lung lavage fluid or whole lungs (Supplemental Figures 1 and 2). Thus, augmenting IL-1RA levels during experimental fungal-associated allergic airway inflammation improves the severity of disease concomitant with lowering type 1 and type 17 responses.

\section{Discussion}

While defining biomarkers of asthma severity, prognosis, and therapeutic responses is a growing area of interest, immunopathogenic pathways contributing to allergic asthma are not well understood. Our previous report on biomarkers in lung lavage fluid identified the IL-1 family members IL-1 $\beta$ and IL-1RA 
as potential contributors to immunopathogenesis vs. immunoprotection during fungal-associated allergic airway inflammation (10). In the current report, analysis of biomarkers in sputum from fungal (+) asthmatics identified IL- $1 \alpha$ and IL-1 $\beta$ as potential immunopathogenic contributors. Previous data from our lab has demonstrated that the level of IL- $1 \alpha$ and IL-1 $\beta$ was associated with the severity of experimental fungal-associated allergic airway inflammation $(10,11)$. Much of what is known regarding the role of IL-1 $\alpha$, IL-1 $\beta$, and IL-1RA in allergic asthma has been derived from studying allergic responses to the model antigen OVA $(22,23)$, rather than aeroallergens such as fungi or house dust mite. The IL-1 family members IL-1 $\beta$ and IL-18 have been posited as therapeutic targets in asthma $(24,25)$, as IL-1 $\beta$ may contribute to steroid resistant asthma (26) and the NLRP3 inflammasome may function in the generation of Th2 cells (27). Despite these observations, it is not well understood how signaling induced by IL-10 and IL-1 $\beta$ contributes to human fungal asthma, and other than observational data, this has not been addressed by our previous experimental studies. Likewise, the significance of elevated IL-1RA in fungal (+) asthmatics (10) and how IL-1RA affects responses induced by IL- $1 \alpha$ and IL-1 $\beta$ signaling are also not clear. Although IL-1 $\beta$ was elevated in both lung lavage fluid and sputum from fungal $(+)$ asthmatics, IL-1 $\alpha$ was elevated in sputum only, whereas IL-1RA was elevated in lung lavage fluid only. While it is perplexing that IL-1 $\alpha$ and IL-1RA were not consistently increased in both human specimens (as observed for IL-1 $\beta$ ), we cannot exclude the possibility that sputum processing with a reducing agent (dithiothreitol) may affect (positively or negatively) the structural integrity and/or detection of some mediators. Nevertheless, as our analysis demonstrates increased levels in lung samples from fungal-sensitized asthmatics, we hypothesize that a balance between pathways induced by IL- $1 \alpha / \mathrm{IL}-1 \beta$ and the regulation of these pathways by IL-1RA determines the severity of fungal asthma.

We and others $(10,11,28-31)$ have employed a live organism, chronic exposure model to identify immunopathogenic processes induced during fungal-associated allergic airway inflammation. This model is relevant because it replicates the daily exposure humans have to fungi such as A. fumigatus (32) and mimics persistent local antigenic stimulation in individuals incapable of adequate fungal clearance (e.g., individuals with SAFS, allergic bronchpulmonary aspergillosis [ABPA]). Key features include induction of type 1, type 2, and type 17 responses; robust neutrophil and eosinophil recruitment; increased IgE production; and AHR. The live organism, chronic fungal-exposure model offers advantages over other models that employ adjuvants to skew immune responses (33) or those that employ commercially available, yet uncharacterized, fungal extract preparations, which have recently come under scrutiny due to their significant variability in allergen content (34). Our initial studies demonstrated that signaling through IL-1R1 had a profound effect on airway and total lung resistance. Better lung function in the absence of the IL-1R1 was associated with histological evidence of decreased mucus in the airways and fewer inflammatory cells, specifically neutrophils and eosinophils. A surprising finding, however, was negligible impact of IL-1R1 deficiency on type 2/Th2 responses. Indeed, the inflammasome component NLRP3 has been shown to bind the IL-4 promoter in CD4 ${ }^{+} \mathrm{T}$ cells (27). Moreover, deficiency in caspase-8, which is required for IL-1 $\beta$ processing, is associated with defective Th2 responses during OVA challenge (23). In contrast, our data demonstrate that, although eosinophil numbers were significantly lower in IL-1R1-deficient mice, the absence of IL-1R1 signaling had no effect on production of the type 2/Th2-associated mediators IL-4, IL-33, CCL11, CCL17, and CCL22 and only a minor effect on IL-5. However, these mediators were measured in either lung homogenates or whole lung digest cell culture supernatants; therefore, we cannot exclude a role for IL-1R1 signaling driving type 2/Th2 responses specifically in $\mathrm{CD}^{+} \mathrm{T}$ cells or ILC2s (despite each of these being present in the lung digest cell culture). In contrast, IL-1R1 signaling was required for the neutrophilic component of fungal-associated allergic airway inflammation. We have reported that IL-1 signaling is critical for the induction of IL-17A and IL-22 after acute $A$. fumigatus challenge (35) and confirmed here that this pathway is also required for IL-17A and IL-22 induction during fungal-associated allergic airway inflammation. Our previous report has shown that IL-22, and likely IL-17A, function in an immunopathogenic capacity during experimental fungal-associated allergic airway inflammation (11). We have recently reported that IL-22 is primarily produced by $\gamma \delta \mathrm{T}$ cells, iNKT cells, and ILC3s during experimental fungal-associated allergic airway inflammation (10), and we speculate these are lung cell sources of IL-17A, as well. Thus, our data imply that IL-1R1 signaling in these cells is required for their ability to produce IL-22 and likely IL-17A. IL-17A — and IL-22, to a lesser extent — is widely recognized as an inducer of mediators that function in neutrophil activation/expansion or recruitment via the induction of neutrophil survival factors and chemoattractants (36). Although we did not observe defective production of the chemoattractants CCL3 and CCL4, we did find a reduction in G-CSF, implying that IL-1R1 signaling 
functions more in neutrophil activation/expansion rather than recruitment. While it would be of interest to interrogate the role of neutrophils in airway hyperreactivity during our live organism fungal asthma mod$\mathrm{el}$, this is not feasible, as the experimental manipulation of neutrophil levels (e.g., depletion) results in the development of invasive fungal infection (37). Two recent studies demonstrated that expression of IL-1R1 in sputum samples predicted airway neutrophilic inflammation and airway obstruction in neutrophilic severe allergic asthma $(38,39)$. Moreover, fungal $\beta$-glucan alone or in combination with LPS can drive a mixed Th2/Th17 response that culminates in a neutrophilic, steroid refractory asthma (40). This is supported by our previous work demonstrating that immunopathogenic inflammatory responses (such as IL- $1 \beta$ production and neutrophil recruitment) during fungal asthma is primarily driven by the fungal $\beta$-glucan receptor Dectin- 1 (11). Collectively, our data lead us to hypothesize that a Dectin-1/IL-1 $\alpha / \mathrm{IL}-1 \beta / \mathrm{IL}-1 \mathrm{R} 1 / \mathrm{IL}-17 \mathrm{~A} / \mathrm{G}-\mathrm{CSF}$ axis supports neutrophilic inflammation during severe fungal-associated allergic airway inflammation.

High serum levels of IL-1RA have been shown to correlate with lower risk of worsening asthma control and attacks in adults (41). In contrast, serum IL-1RA may be elevated during acute asthma exacerbations in a pediatric cohort compared with those with stable asthma (42). Interestingly, this study also documented that IL-1RA decreased in response to corticosteroids. An additional study has shown that patients with neutrophilic asthma have an impairment in the ratio of IL-1RA/IL-1 $\beta$ when compared with eosinophilic asthma (43). Our previous study reported that IL-1RA was increased in human asthmatics sensitized to fungi and was negatively associated with the lung function measurement PC20 (10). Collectively, data in humans have not provided a clear insight into the role of IL-1RA in allergic asthma. In experimental studies, exposing mice deficient in IL-1RA to the OVA asthma model resulted in worse lung function and enhanced type $2 / \mathrm{Th} 2$ responses (22). In contrast to this observation, we did not observe enhanced type 2/Th2 responses when mice deficient in IL-1RA were subjected to fungal-associated allergic airway inflammation. In fact, despite observing higher IL-33 levels in lung homogenates, IL-4 and IL-5 production by lung digest cells were significantly reduced. Additionally, the major chemoattractant for eosinophils, CCL11, was also significantly reduced, and the number of eosinophils trended lower. However, histological assessment demonstrated a lack of mucus production and lower Muc5ac and Gob5 mRNA expression, which was associated with the lower type 2 responses observed. Instead, histology revealed an unrestricted inflammatory process occurring in the absence of IL-1RA. Based on flow cytometry data, this inflammation was neutrophilic in nature. A component of IL-1RA-mediated regulation was at the level of IL-17A, which was produced at 4-fold higher levels in IL-1RA-deficient mice compared with WT controls. This elevation in IL-17A was further associated with heightened induction of G-CSF, as well as CCL3 and CCL4. Although data exist showing that mice deficient in IL-1RA demonstrate baseline increases in inflammatory responses in models of arthritis and psoriasis $(44,45)$, this has been exclusively observed in IL-1RA deficiency on the Balb/c background and not in those on the C57BL/6 background, which we employed here. Collectively, these data lead us to hypothesize that a primary function of IL-1RA is to restrict the magnitude of IL-17A-associated responses that promote neutrophilic inflammation during fungal-associated allergic airway inflammation.

Growing evidence supports a role for a type $1 / \mathrm{Th} 1 / \mathrm{IFN}-\gamma$ signature in severe asthma associated with corticosteroid resistance. Type $1 / \mathrm{Th} 1 / \mathrm{IFN}-\gamma$-associated immune responses are exacerbated in the airways of individuals with severe asthma (17). Modeling this in an experimental severe asthma model (albeit not fungal) shows that mice deficient in IFN- $\gamma$ fail to exhibit AHR (17). More recently, severe asthmatics demonstrate high mRNA levels of the type 1/Th1-associated chemokine CXCL10 in BAL cells (18). In an experimental asthma model, IFN- $\gamma$ and CXCL10 were found to be refractory to corticosteroid treatment. Interestingly, high CXCL10 gene expression was also associated with a mast cell signature in both humans and mice (18). Our data provide further insight into this pathway, as IFN- $\gamma$ and its associated chemokines CXCL9 and CXCL10 were dependent on IL-1R1 signaling for optimal production during fungal-associated allergic airway inflammation. While IL-1R1 dependency for IL-17A/IL-22 responses was expected, IL-1R1-dependent IFN- $\gamma$ production was a surprising finding. Although we have reported that iNKT cells produce IL-22 (and likely IL-17A) in an IL-1R1-dependent manner after acute and chronic A. fumigatus exposure (10, 46), iNKT cells have also been reported to be a source of IFN- $\gamma$ after $A$. fumigatus exposure $(47,48)$. The dependency of IFN- $\gamma$ production on IL-1R1 is not widely recognized, with only a few reports showing IL-1R1dependent IFN- $\gamma$ production by $\mathrm{CD}^{+} \mathrm{T}$ cells during contact hypersensitivity (49) and cardiac transplantation (50). In contrast, IL-1RA functioned as a potent regulator of IFN- $\gamma$ production during fungal-associated allergic airway inflammation. Moreover, IL-1RA-mediated regulation of IFN- $\gamma$ has been shown in models 


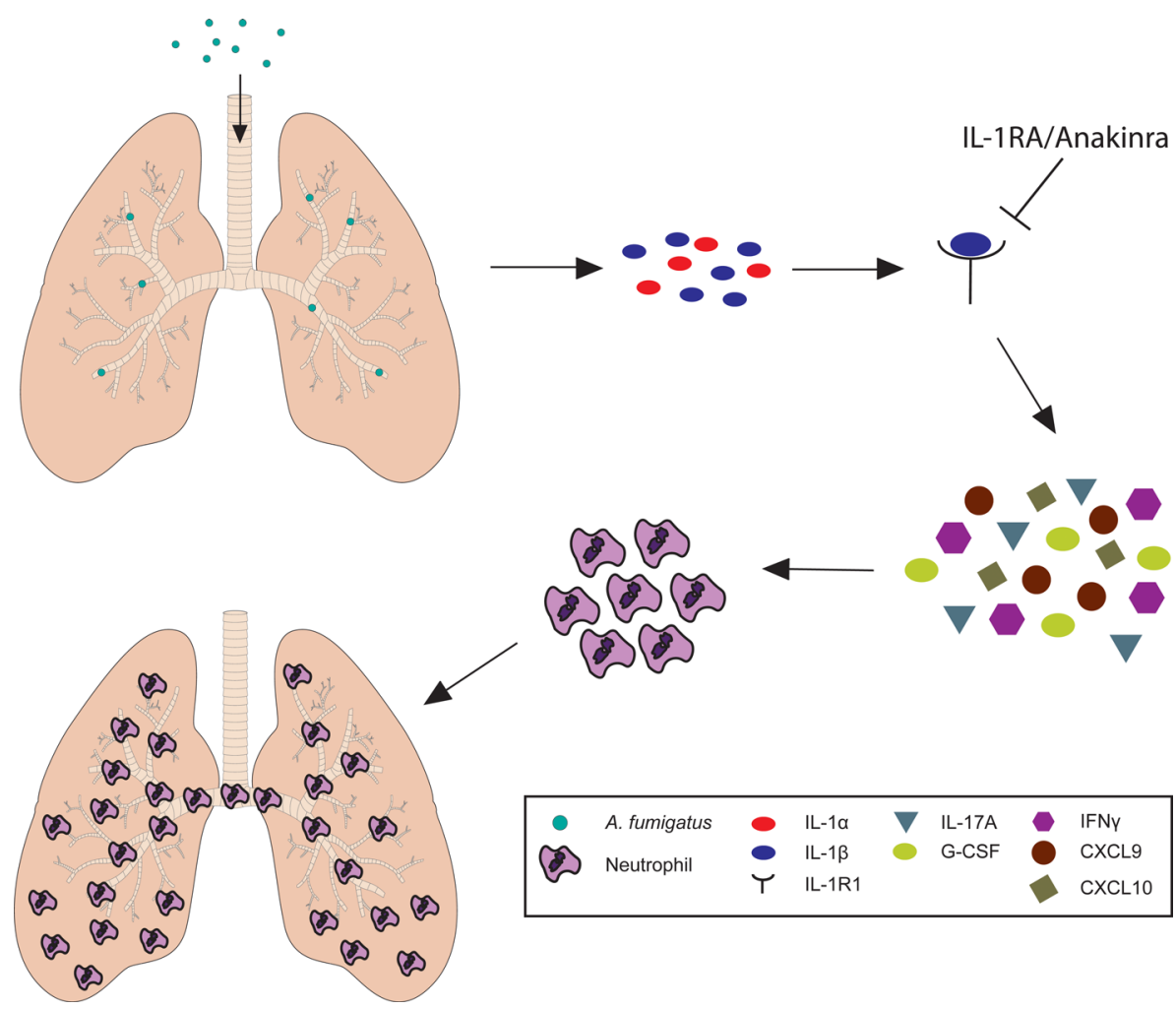

Figure 9. Impact of IL-1R1 signaling during experimental fungal-associated allergic airway inflammation. Chronic exposure to live airborne $A$. fumigatus conidia induces IL-1 $\alpha$ and IL-1 $1 \beta$ production and subsequent signaling via IL-1R1. IL-1R1 signaling induces the production of type 1 and type 17 responses, which augments neutrophil recruitment to the lung. Enhanced neutrophil recruitment negatively impacts both central and peripheral airway function. The IL-1R1 antagonist IL-1RA is essential for regulating the magnitude of IL-1R1 signaling, and augmenting IL-1RA levels result in lower type 1 and type 17 responses and improved lung function.

of Toxoplasma gondii-mediated arthritis (51) and experimental autoimmune encephalomyelitis (52). IL-1RA is required for the induction of IFN- $\gamma$ during cutaneous Leishmania major infection (53). Other studies have shown that treatment of human PBMCs with IL-1RA had no effect on Staphylococcus epidermidis-induced IFN- $\gamma$ production (54). Overall, these observations suggest that the interaction/relationship between IL-1RA and IFN- $\gamma$ is diverse based on the model system employed.

The final question we asked was whether the IL-1R1/IL-1RA axis was a viable therapeutic target in fungal-associated allergic airway inflammation. Human recombinant IL-1RA, Kineret/anakinra, is US Food and Drug Administration-approved (FDA-approved) for the treatment of rheumatoid arthritis and neonatal onset multisystem inflammatory disease (NOMID), a subtype of cryopyrin-associated periodic syndromes (CAPS). We show that treating mice with Kineret/anakinra over the development of fungal-associated allergic airway inflammation was effective at improving lung function. Kineret/anakinra lowered both AHR (Newtonian resistance), which is the resistance of the central or conducting airways, and total lung resistance, which is the level of constriction in the lungs. A previous study has demonstrated that Kineret/anakinra administered during invasive aspergillosis resulted in reduced IL-17A production and increased IFN- $\gamma$ production in $p 47$ phox ${ }^{-1}$ mice (i.e., chronic granulomatous disease mice), while treatment of WT mice resulted in the opposite effect: decreased IFN- $\gamma$ and no effect of IL-17A (55). A second study has demonstrated that treating mice deficient in CFTR with Kineret/anakinra lowered $A$. fumigatus lung burden. However, the only immunologic mechanism proposed in this study was lower IL-1 $\beta$ production (56). Finally, a study using PBMCs from patients with allergic bronchopulmonary aspergillosis stimulated with heat-killed $A$. fumigatus and cultured with etanercept, adalimumab, or Kineret/anakinra demonstrated lower IL-5 and IL-13 production, although other mediators such as IL-17A and IFN- $\gamma$ were not investigated (57). Our study is the first to our knowledge to document efficacy of blocking IL-1R1 signaling in vivo during experimental aeroallergen-associated allergic airway inflammation. We show that this was effective at lowering both type 1 (IFN- $\gamma$ ) and type 17 responses (IL-17A), but blocking IL-1R1 sig- 
naling had no effect on modulating type 2 responses or inflammatory cell recruitment.

In summary, we have identified IL-1 family members as putative biomarkers of disease severity in human asthmatics that were sensitized the fungi. Interrogating the role of IL-1R1 signaling in experimental fungalassociated allergic airway inflammation revealed this pathway to drive the induction of type 1 and type 17 responses that augmented neutrophilic responses, leading to worse lung function. In turn, IL-1RA was required to regulate the magnitude of type 1 and type 17 responses, neutrophilic inflammation, and lung function during experimental fungal-associated allergic airway inflammation (Figure 9). IL-1RA itself also demonstrated an ability to serve as a viable target for treating fungal-associated allergic airway inflammation. Surprisingly, IL-1R1/IL-1RA had varied or little impact on type 2 responses. Collectively, our results provide insight into the role that IL-1 family members play in immunopathogenesis during severe allergic asthma.

\section{Methods}

Subjects, sputum induction and processing, and Luminex analysis. Patients with mild to severe asthma were comprehensively characterized according to the NHLBI SARP phenotype protocol at Wake Forest School of Medicine as previously described (58), and a description of this cohort was recently reported (59). The sputum induction method was adopted from the NHLBI Asthma Clinical Research Network and used in SARP (60). BALF and sputum samples were derived from both the SARP 1 and 2 cohorts. The number of subjects who had both BALF and sputum collected in the current cohort was $N=21$ (18\% of the subjects in Table 1). Per SARP protocol, BALF and sputum were not collected within a week of each other. Sputum was processed immediately after collection, and cell cytospins were stained for differential counts of at least 500 nonsquamous cells/subject slide. Total WBC count in sputum was determined via trypan blue staining and enumeration using a hemacytometer. Cell-free supernatants were aliquoted and stored at $-80^{\circ} \mathrm{C}$ before use in Luminex analyses (see below). Biospecimens were randomly selected without a priori selection based on asthma severity, rather than based on whether they were skin test-positive or -negative for Alternaria, Aspergillus, and/or Cladosporium. Sputum supernatants were assayed for different inflammatory cytokine, chemokine, and growth factor protein concentrations using Milliplex Human Cytokine/Chemokine Panels I, II, III, and IV (catalogs HCYTOMAG-60K-PX41, HCYP2MAG-62K-PX23, HCYP3MAG-63K, and HCY4MG-64K-PX21, respectively; MilliporeSigma). Standards for determination of linear curve plus 2 control samples representing high and low levels of each cytokine/chemokine were included in each assay.

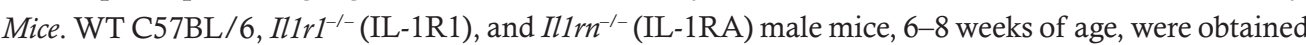
from The Jackson Laboratory.

A. fumigatus preparation and fungal-associated allergic airway inflammation model. A. fumigatus isolate 13073 (ATCC) was maintained on potato dextrose agar for $5-7$ days at $37^{\circ} \mathrm{C}$. Conidia were harvested by washing the culture flask with $50 \mathrm{~mL}$ of sterile PBS supplemented with $0.1 \%$ Tween 20 . The conidia were then passed through a sterile $40-\mu \mathrm{m}$ nylon membrane to remove hyphal fragments and were enumerated on a hemacytometer. The repeated $A$. fumigatus exposure model was employed as previously described (11). Briefly, mice were lightly anesthetized with isoflurane and administered $1 \times 10^{7}$ live A. fumigatus conidia in a volume of $50 \mu \mathrm{L}$ of PBS intratracheally (i.t.). After resting for 7 days, mice were challenged i.t. with $1 \times 10^{6}$ live $A$. fumigatus conidia in $50 \mu \mathrm{L}$ of PBS daily for 5 consecutive days (days 7, 8, 9, 10, and 11), allowed to rest for 2 consecutive days (days 12 and 13), and then challenged i.t. with $1 \times 10^{6}$ live A. fumigatus conidia in $50 \mu \mathrm{L}$ of PBS daily for 3 consecutive days (days 14, 15 and 16). Twenty-four hours after the last $A$. fumigatus challenge (day 17), mice were sacrificed for analysis via ketamine/xylazine (Vet One) overdose and aortic exsanguination.

Whole lung cytokine and chemokine analysis, lung cell isolation, and culture. Following sacrifice, the right lung was homogenized in PBS supplemented with Complete Mini protease inhibitor tablets (Roche Diagnostics), clarified by centrifugation $\left(12,000 \mathrm{~g}\right.$ for 10 minutes), and stored at $-80^{\circ} \mathrm{C}$. From lung homogenate supernatants, IL-33, CCL17, and CCL22 levels were quantified by ELISA (R\&D Systems). For lung cell isolation, the lungs were collected and minced in IMDM media (MilliporeSigma) supplemented with $1 \times$ penicillin-streptomycin-glutamine (Mediatech), 10\% heat-inactivated FBS (Invitrogen), and $0.4 \mathrm{mg} / \mathrm{mL}$ polymyxin B (Thermo Fisher Scientific), followed by incubation for 60 minutes with tissue culture grade type IV collagenase $(1 \mathrm{mg} / \mathrm{mL}$; MiiliporeSig$\mathrm{ma})$ in a $37^{\circ} \mathrm{C}$ orbital shaker at $100 \mathrm{rpm}$. The cell suspension was filtered through sterile $70-\mu \mathrm{m}$ and $40-\mu \mathrm{m}$ nylon filters, and RBCs were lysed with ACK buffer (Lonza) to create single-cell preparations. One million cells in a volume of $200 \mu \mathrm{L}$ were cultured overnight with 1 million A. fumigatus conidia (1:1), followed by collection and clarification of supernatants. Supernatants from lung digest cells 
were analyzed for protein levels of 32 cytokines and chemokines using the Luminex-based Milliplex multiplex suspension cytokine array (MilliporeSigma), according to the manufacturer's instructions. The data were analyzed using Bio-Plex Manager software (Bio-Rad). Lung digest cell supernatants were also used to quantify levels of IL-22 by ELISA (R\&D Systems).

Lung cell flow cytometry. Lung cells were isolated via BAL or by enzymatic digestion of whole lungs as previously described $(11,16)$. Cells were washed, and Fc receptors were blocked with Mouse BD Fc Block (BD Biosciences) at $4^{\circ} \mathrm{C}$ for 20 minutes. Thereafter, cells were stained with a single-color LIVE/DEAD Fixable Dead Cell Stain (Invitrogen), followed by labeling with specific immune cell surface markers. The following staining parameters were employed: eosinophils as $\mathrm{CD} 45^{+} \mathrm{CD} 11 \mathrm{~b}^{+}$Siglec $\mathrm{F}^{+} \mathrm{Ly}-6 \mathrm{G}^{-}$using anti-CD45 (BioLegend, catalog 103115, clone 30-F11), anti-CD11b (BioLegend, catalog 101237, clone M1/70), anti-Siglec F (BioLegend, catalog 155507, clone S17007L), and anti-Ly-6G (BioLegend, catalog 127621, clone 1A8); neutrophils as $\mathrm{CD}_{4} 5^{+} \mathrm{CD} 11 \mathrm{~b}^{+} \mathrm{Ly} 6 \mathrm{G}^{+}$Siglec $\mathrm{F}^{-}$using anti-CD45, anti CD11b, anti-Ly-6G, and anti-Siglec F; CD4 ${ }^{+} \mathrm{T}$ cells as $\mathrm{CD}^{+} 5^{+} \mathrm{CD}^{+} \mathrm{CD}^{+}$using anti-CD45, anti-CD4 (BioLegend catalog 100421, clone GK1.5), and anti-CD3 ${ }^{+}$ (BioLegend catalog 100216, clone 17A2); $\gamma \delta$ T cells as $\mathrm{CD}^{+} 5^{+} \gamma \delta \mathrm{TCR}^{+} \mathrm{CD}^{+}$using anti-CD45, anti- $\gamma \delta \mathrm{TCR}^{+}$ (BioLegend catalog 118117, clone GL3), and anti-CD3; and iNKT cells as TCR $\beta^{+}$PBS-57 CD1d tetramer using anti-TCR $\beta$ (BioLegend catalog 109239, clone H57-597) and anti-PBS-57 CD1d tetramer ${ }^{+}$(NIH Tetramer Core, Emory University, Atlanta, Georgia, USA) Samples were acquired using a 4-laser, 20-parameter analytic BD LSRFortessa, and data were analyzed using FlowJo software (Tree Star Inc.). Unstained lung leukocytes served as a control for background fluorescence and gating, as well as single-color controls. Appropriately stained UltraComp eBeads (Thermo Fisher Scientific) served as single-color controls.

Pulmonary function assessment. Individual anesthetized $A$. fumigatus-exposed mice were intubated, and each animal was attached to a computer-controlled volume ventilator (flexiVent; SCIREQ). Regular breathing was set at 150 breaths per minute, with volume and pressure controlled by the flexiVent system based on individual animal weights. Positive end-expiratory pressure was set to $2 \mathrm{~cm}_{2} \mathrm{H}_{2}$ and measured during each breath stroke. The single-frequency forced oscillation technique was employed to measure total/dynamic lung resistance $(R)$ and compliance $(C)$. The low-frequency/broadband forced oscillation technique was employed to measure Newtonian resistance ( $\mathrm{Rn}$; also known as airway hyperreactivity), tissue damping $(\mathrm{G})$, and tissue elastance $(\mathrm{H})$. All measurements were collected at baseline and after a linear dose response with methacholine challenge $(10-40 \mathrm{mg} / \mathrm{mL})$, as previously described $(10,16)$. Lung function was also assessed in naive WT and mutant mice, which confirmed no baseline anomalies and no differences between groups, as we have previously reported (11).

In vivo treatment with Kineret/anakinra. In specific experiments, WT C57BL/6 mice were treated with Kineret/anakinra (SOBI), a recombinant, nonglycosylated form of human IL-1RA. Specifically, mice were sensitized and challenged with A. fumigatus as described above and i.p. injected with Kineret/anakinra (10 $\mathrm{mg} / \mathrm{kg}$ or $50 \mathrm{mg} / \mathrm{kg}$ in $200 \mu \mathrm{L} 0.9 \% \mathrm{NaCL}$; R\&D Systems) or vehicle daily on days $0-16$. Twenty-four hours after final injection and $A$. fumigatus challenge, mice underwent pulmonary function assessment or cytokine/chemokine analysis as described above.

Statistics. Data were analyzed using GraphPad Prism Version 7.0 statistical software (GraphPad Software) Comparisons between groups when data were normally distributed were made with the 2-tailed unpaired Student's $t$ test or 2-way-ANOVA. Significance was accepted at a value of $P<0.05$.

Study approval. Human subjects were enrolled with informed consent under approved Wake Forest School of Medicine IRB BG01-425 (Wake Forest University IRB). Human samples were analyzed under UAB IRB X130827009 (UAB IRB for Human Use). All animal research was conducted under approved UAB IACUC protocols 10199 and 20236 (UAB IACUC) and approved by Tulane IACUC protocols 217 and 412 (Tulane IACUC). All animals were housed in a specific pathogen-free, Association for Assessment and Accreditation of Laboratory Animal Care-certified facility and handled according to NIH Public Health Service Office of Guide for the Care and Use of Laboratory Animals (National Academies Press, 2011).

\section{Author contributions}

MSG and CS designed the research. MSG, KMR, JMG, JPB, and MJ performed the research. ZY and SM performed the lung function analysis. MSG and CS analyzed the data. ATH and DAM conducted all human subject recruitment and clinical analysis. MSG and CS wrote the manuscript. CS supervised all aspects of the project. All authors agreed to all of the content of the submitted manuscript. 


\section{Acknowledgments}

This work was supported was supported by Public Health Service grants HL109164 (DA), HL122426 (CS), and HL136211 (CS).

Address correspondence to: Chad Steele, Department of Microbiology and Immunology, School of Medicine, Tulane University, 1430 Tulane Avenue, Room 5053, New Orleans, Louisiana 70112, USA. Phone: 504.988.5386; Email: csteele4@tulane.edu.

1. Moore WC, et al. Characterization of the severe asthma phenotype by the National Heart, Lung, and Blood Institute's Severe Asthma Research Program. J Allergy Clin Immunol. 2007;119(2):405-413.

2. Schatz M, Rosenwasser L. The allergic asthma phenotype. J Allergy Clin Immunol Pract. 2014;2(6):645-649.

3. Denning DW, O'Driscoll BR, Hogaboam CM, Bowyer P, Niven RM. The link between fungi and severe asthma: a summary of the evidence. Eur Respir J. 2006;27(3):615-626.

4. Medrek SK, Kao CC, Yang DH, Hanania NA, Parulekar AD. Fungal Sensitization Is Associated with Increased Risk of Life-Threatening Asthma. J Allergy Clin Immunol Pract. 2017;5(4):1025-1031.e2.

5. Agarwal R. Severe asthma with fungal sensitization. Curr Allergy Asthma Rep. 2011;11(5):403-413.

6. Masaki K, et al. Characteristics of severe asthma with fungal sensitization. Ann Allergy Asthma Immunol. 2017;119(3):253-257.

7. Castanhinha S, et al. Pediatric severe asthma with fungal sensitization is mediated by steroid-resistant IL-33. J Allergy Clin Immunol. 2015;136(2):312-22.e7.

8. Durham AL, Caramori G, Chung KF, Adcock IM. Targeted anti-inflammatory therapeutics in asthma and chronic obstructive lung disease. Transl Res. 2016;167(1):192-203.

9. Overton NL, Simpson A, Bowyer P, Denning DW. Genetic susceptibility to severe asthma with fungal sensitization. Int J Immunogenet. 2017;44(3):93-106.

10. Reeder KM, et al. The common $\gamma$-chain cytokine IL-7 promotes immunopathogenesis during fungal asthma. Mucosal Immunol. 2018;11(5):1352-1362.

11. Lilly LM, et al. The $\beta$-glucan receptor dectin-1 promotes lung immunopathology during fungal allergy via IL-22. J Immunol. 2012;189(7):3653-3660.

12. Ray A, Kolls JK. Neutrophilic Inflammation in Asthma and Association with Disease Severity. Trends Immunol. 2017;38(12):942-954.

13. Barnes PD, Marr KA. Risks, diagnosis and outcomes of invasive fungal infections in haematopoietic stem cell transplant recipients. Br J Haematol. 2007;139(4):519-531.

14. Hartl D, Latzin P, Zissel G, Krane M, Krauss-Etschmann S, Griese M. Chemokines indicate allergic bronchopulmonary aspergillosis in patients with cystic fibrosis. Am J Respir Crit Care Med. 2006;173(12):1370-1376.

15. Latzin P, Hartl D, Regamey N, Frey U, Schoeni MH, Casaulta C. Comparison of serum markers for allergic bronchopulmonary aspergillosis in cystic fibrosis. Eur Respir J. 2008;31(1):36-42.

16. Garth JM, et al. Acidic Mammalian Chitinase Negatively Affects Immune Responses during Acute and Chronic Aspergillus fumigatus Exposure. Infect Immun. 2018;86(7):e00944-17.

17. Raundhal M, et al. High IFN- $\gamma$ and low SLPI mark severe asthma in mice and humans. J Clin Invest. 2015;125(8):3037-3050.

18. Gauthier M, et al. Severe asthma in humans and mouse model suggests a CXCL10 signature underlies corticosteroid-resistant Th1 bias. JCI Insight. 2017;2(13):94580.

19. Mayer-Barber KD, Yan B. Clash of the Cytokine Titans: counter-regulation of interleukin-1 and type I interferon-mediated inflammatory responses. Cell Mol Immunol. 2017;14(1):22-35.

20. Satoh T, Otsuka A, Contassot E, French LE. The inflammasome and IL-1 $\beta$ : implications for the treatment of inflammatory diseases. Immunotherapy. 2015;7(3):243-254.

21. Jesus AA, Goldbach-Mansky R. IL-1 blockade in autoinflammatory syndromes. Annu Rev Med. 2014;65:223-244.

22. Nakae S, et al. IL-1 is required for allergen-specific Th2 cell activation and the development of airway hypersensitivity response. Int Immunol. 2003;15(4):483-490.

23. Qi X, et al. Critical role of caspase-8-mediated IL-1 signaling in promoting Th2 responses during asthma pathogenesis. Mucosal Immunol. 2017;10(1):128-138.

24. Xiao Y, Xu W, Su W. NLRP3 inflammasome: A likely target for the treatment of allergic diseases. Clin Exp Allergy. 2018;48(9):1080-1091.

25. Liao Z, et al. IL-1ß: a key modulator in asthmatic airway smooth muscle hyper-reactivity. Expert Rev Respir Med. 2015;9(4):429-436.

26. Kim RY, et al. Role for NLRP3 Inflammasome-mediated, IL-1 $\beta$-Dependent Responses in Severe, Steroid-Resistant Asthma. Am J Respir Crit Care Med. 2017;196(3):283-297.

27. Bruchard M, et al. The receptor NLRP3 is a transcriptional regulator of TH2 differentiation. Nat Immunol. 2015;16(8):859-870.

28. Fei M, et al. TNF-alpha from inflammatory dendritic cells (DCs) regulates lung IL-17A/IL-5 levels and neutrophilia versus eosinophilia during persistent fungal infection. Proc Natl Acad Sci USA. 2011;108(13):5360-5365.

29. Murdock BJ, et al. Coevolution of $\mathrm{TH} 1, \mathrm{TH} 2$, and $\mathrm{TH} 17$ responses during repeated pulmonary exposure to Aspergillus fumigatus conidia. Infect Immun. 2011;79(1):125-135.

30. Shreiner AB, et al. Repeated exposure to Aspergillus fumigatus conidia results in CD4+ T cell-dependent and -independent pulmonary arterial remodeling in a mixed Th1/Th2/Th17 microenvironment that requires interleukin-4 (IL-4) and IL-10. Infect Immun. 2012;80(1):388-397.

31. Murdock BJ, et al. Interleukin-17 drives pulmonary eosinophilia following repeated exposure to Aspergillus fumigatus conidia. Infect Immun. 2012;80(4):1424-1436. 
32. Kwon-Chung KJ, Sugui JA. Aspergillus fumigatus--what makes the species a ubiquitous human fungal pathogen? PLoS Pathog. 2013;9(12):e1003743.

33. Hogaboam CM, et al. Chronic airway hyperreactivity, goblet cell hyperplasia, and peribronchial fibrosis during allergic airway disease induced by Aspergillus fumigatus. Am J Pathol. 2000;156(2):723-732.

34. Wurth MA, et al. Human IgE mAbs define variability in commercial Aspergillus extract allergen composition. JCI Insight. 2018;3(20):123387.

35. Garth JM, et al. IL-33 Signaling Regulates Innate IL-17A and IL-22 Production via Suppression of Prostaglandin $\mathrm{E}_{2}$ during Lung Fungal Infection. J Immunol. 2017;199(6):2140-2148.

36. Aujla SJ, et al. IL-22 mediates mucosal host defense against Gram-negative bacterial pneumonia. Nat Med. 2008;14(3):275-281.

37. Stephens-Romero SD, Mednick AJ, Feldmesser M. The pathogenesis of fatal outcome in murine pulmonary aspergillosis depends on the neutrophil depletion strategy. Infect Immun. 2005;73(1):114-125.

38. Evans MD, Esnault S, Denlinger LC, Jarjour NN. Sputum cell IL-1 receptor expression level is a marker of airway neutrophilia and airflow obstruction in asthmatic patients. J Allergy Clin Immunol. 2018;142(2):415-423.

39. Rossios C, et al. Sputum transcriptomics reveal upregulation of IL-1 receptor family members in patients with severe asthma. J Allergy Clin Immunol. 2018;141(2):560-570.

40. Zhang $\mathrm{Z}$, et al. $\beta$-Glucan exacerbates allergic asthma independent of fungal sensitization and promotes steroid-resistant $\mathrm{T}_{\mathrm{H}} 2 /$ $\mathrm{T}_{\mathrm{H}} 17$ responses. J Allergy Clin Immunol. 2017;139(1):54-65.e8.

41. Akiki Z, et al. Serum cytokine profiles as predictors of asthma control in adults from the EGEA study. Respir Med. 2017;125:57-64

42. Kato M, Yamada Y, Maruyama K, Hayashi Y. Serum eosinophil cationic protein and 27 cytokines/chemokines in acute exacerbation of childhood asthma. Int Arch Allergy Immunol. 2010;152 Suppl 1:62-66.

43. Gao P, et al. Anti-inflammatory deficiencies in neutrophilic asthma: reduced galectin-3 and IL-1RA/IL-1ß. Respir Res. 2015;16:5.

44. Horai R, et al. Development of chronic inflammatory arthropathy resembling rheumatoid arthritis in interleukin 1 receptor antagonist-deficient mice. J Exp Med. 2000;191(2):313-320.

45. Shepherd J, Little MC, Nicklin MJ. Psoriasis-like cutaneous inflammation in mice lacking interleukin-1 receptor antagonist. J Invest Dermatol. 2004;122(3):665-669.

46. Reeder KM, et al. Role of Common $\gamma$-Chain Cytokines in Lung Interleukin-22 Regulation after Acute Exposure to Aspergillus fumigatus. Infect Immun. 2018;86(10):e00157-18.

47. Cohen NR, et al. Innate recognition of cell wall $\beta$-glucans drives invariant natural killer T cell responses against fungi. Cell Host Microbe. 2011;10(5):437-450.

48. Albacker LA, et al. Invariant natural killer T cells recognize a fungal glycosphingolipid that can induce airway hyperreactivity. Nat Med. 2013;19(10):1297-1304.

49. Kish DD, Gorbachev AV, Fairchild RL. IL-1 receptor signaling is required at multiple stages of sensitization and elicitation of the contact hypersensitivity response. J Immunol. 2012;188(4):1761-1771.

50. Iida S, et al. IL-1 Receptor Signaling on Graft Parenchymal Cells Regulates Memory and De Novo Donor-Reactive CD8 T Cell Responses to Cardiac Allografts. J Immunol. 2016;196(6):2827-2837.

51. Washino T, Moroda M, Iwakura Y, Aosai F. Toxoplasma gondii infection inhibits Th17-mediated spontaneous development of arthritis in interleukin-1 receptor antagonist-deficient mice. Infect Immun. 2012;80(4):1437-1444.

52. Matsuki T, Nakae S, Sudo K, Horai R, Iwakura Y. Abnormal T cell activation caused by the imbalance of the IL-1/ IL-1R antagonist system is responsible for the development of experimental autoimmune encephalomyelitis. Int Immunol. 2006;18(2):399-407.

53. Kautz-Neu K, Kostka SL, Dinges S, Iwakura Y, Udey MC, von Stebut E. A role for leukocyte-derived IL-1RA in DC homeostasis revealed by increased susceptibility of IL-1RA-deficient mice to cutaneous leishmaniasis. J Invest Dermatol. 2011;131(8):1650-1659.

54. Stuyt RJ, et al. Regulation of Staphylococcus epidermidis-induced IFN-gamma in whole human blood: the role of endogenous IL-18, IL-12, IL-1, and TNF. Cytokine. 2003;21(2):65-73.

55. de Luca A, et al. IL-1 receptor blockade restores autophagy and reduces inflammation in chronic granulomatous disease in mice and in humans. Proc Natl Acad Sci USA. 2014;111(9):3526-3531.

56. Iannitti RG, et al. IL-1 receptor antagonist ameliorates inflammasome-dependent inflammation in murine and human cystic fibrosis. Nat Commun. 2016;7:10791

57. Becker KL, et al. Pattern recognition pathways leading to a Th2 cytokine bias in allergic bronchopulmonary aspergillosis patients. Clin Exp Allergy. 2015;45(2):423-437.

58. Moore WC, et al. Identification of asthma phenotypes using cluster analysis in the Severe Asthma Research Program. Am J Respir Crit Care Med. 2010;181(4):315-323.

59. Hastie AT, et al. Complex association patterns for inflammatory mediators in induced sputum from subjects with asthma. Clin Exp Allergy. 2018;48(7):787-797.

60. Moore WC, et al. Sputum neutrophil counts are associated with more severe asthma phenotypes using cluster analysis. $J$ Allergy Clin Immunol. 2014;133(6):1557-63.e5 